\title{
dev 71965
}

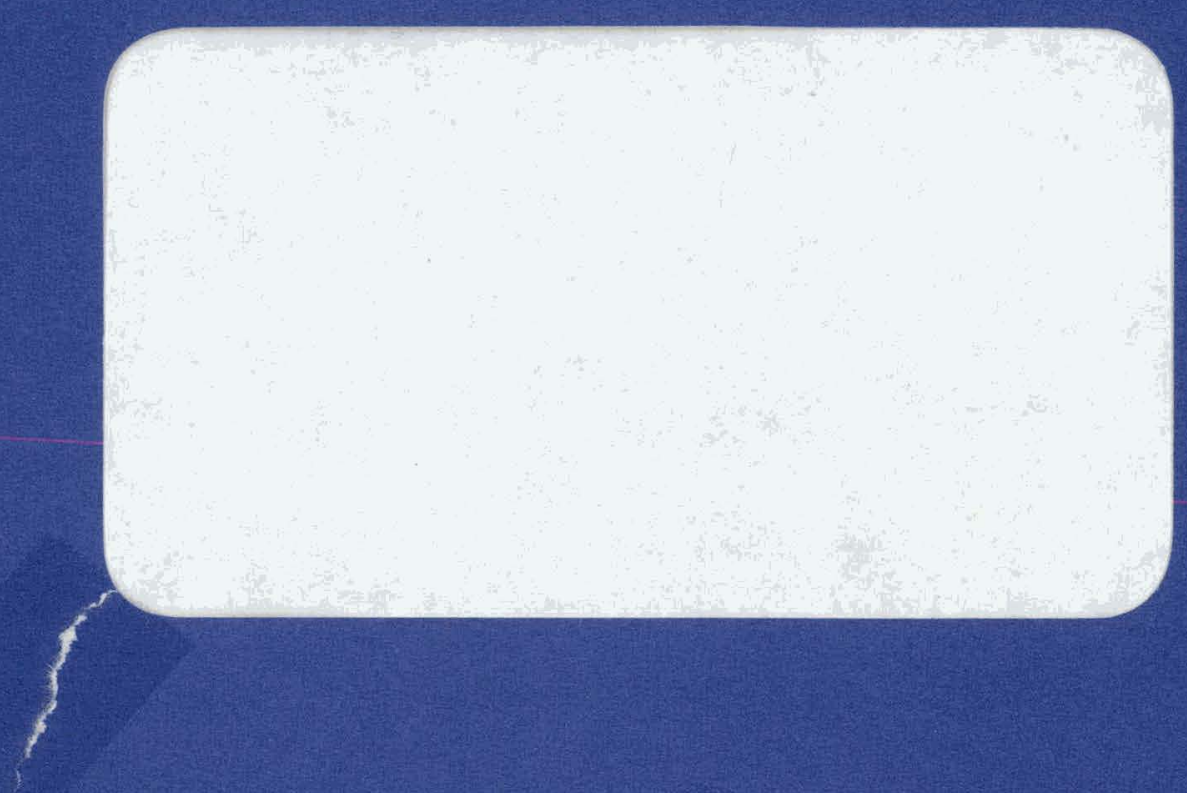

PATEMT CLEARANCE OPTAMEE, RELEASE TN

Westinghouse Atomic Power Division

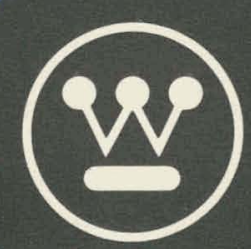




\section{DISCLAIMER}

This report was prepared as an account of work sponsored by an agency of the United States Government. Neither the United States Government nor any agency Thereof, nor any of their employees, makes any warranty, express or implied, or assumes any legal liability or responsibility for the accuracy, completeness, or usefulness of any information, apparatus, product, or process disclosed, or represents that its use would not infringe privately owned rights. Reference herein to any specific commercial product, process, or service by trade name, trademark, manufacturer, or otherwise does not necessarily constitute or imply its endorsement, recommendation, or favoring by the United States Government or any agency thereof. The views and opinions of authors expressed herein do not necessarily state or reflect those of the United States Government or any agency thereof. 


\section{DISCLAIMER}

Portions of this document may be illegible in electronic image products. Images are produced from the best available original document. 
THEORETICAL CALCULATIONS OF INELASTIC SCATTERTNG

MATRICES FOR MUTIGROUP REACTOR-ANAIYYSIS,

C. P. Bhalla

Reactor Development

September 1964

Prepared for the United States Atomic Energy Commission New York Operations Office

Under Contract AT(30-1) -3269

(Sub-task LRD -20I)

WESTINGHOUSE ELECTRIC CORPORATION

Atomic Power Division

P. 0. Box 355

Pittsburgh, Pennsylvania 15230 
This report was prepared as an account of Government sponsored work. Neither the United States, nor the Commission, nor any person acting on behalf of the Commission:

A. Makes any warranty or representation, expressed or impilied, with réspect to the accuracy, completeness, or usefulness of the information contained in this report, or that the usc of any information, apparatus, method, or process disclosed in this report may not infringe privately owned rights; or

B. Assumes any liabilities with respect to the use of, or for damages resulting from the use of any information, apparatus, method, or process disclosed in this report.

As used in the above, "person aoting on behalf of tho Commicoion" includes any employe or contractor of the Commission, or employe of such contractor, to the extent that such employe or contractor of the Commission, or employe of such contractor prepares, disseminates, or provides access to, any information pursuant to his employment or contract with the Commission, or his employment with such contractor. 
USAEC, New York Operations Office; 376 Hudson St., New York 14, N.Y.

Director, Reactor Development Division

USAEC, Resident Engineer, Westinghouse Atomic Power Division

USAEC, Division of Reactor Development and Technology,

Washington 25, D. C. Attn: W. R. Voigt

USAEC, Division of Technical Information, P. 0. Box 62, Oak Ridge, Tennessee

USAEC, Assistant General Counsel for Patents, Gernantown, Maryland

Attn: R. A. Andersor

General Electric Atomic Power Eguipment Dept., 2151 South First St., 2

San Jose, California, Attn: Aileen Thompson

The Babcock \& Wilcox Co., Atomic Energy Divislon, 1201 Kemper St.,

P. 0. Box 1260, Lynchburg, Virginia, Attn: J. W. Landis

Allis-Chalmers Manufacturing Co., Nuclear Power Dept. - Greendale, 2

P. O. Box 512, Milwaukee 1, Wisconsin, Attn: C. B. Graham

Combustion Engineering; Inc., Nuclear Division, Windsor, Connecticut, 2

Attn: A. F. Miller.

Hanford Atomic Products Operations, General Electric Co., Richland,

Washington, Attn: W. S. Dowis

Argonne National Laboratory, P. 0. Box 299, Lemont, Iilino1s,

Attn: A. V. Crewe

Yankee Atomic Electric Co., 441 Stuart St., Boston 16, Massachusetts, I

Attn: R. J. Coe

Yankee Atomic Electric Co., 441 Stuart St., Boston 16, Massachusetts, 1

Attn: D. E. Vandenburgh

Los Angeles Dept. of Water \& Power, 207 South Broadway, P. 0. Box 3669, 1 Terminal Annex, Los Angeles 54, California, Attn: S. B. Nelson

Connecticut Yankee Atomic Power Co., P. O. Box 2010, Hartford 1,

Connecticut, Attn: S. R. Knapp

Southern California Édison Co., P. O. Box 351, Lus Angeles 53,

California, Attn: J. F. Davenport

Southern California Edison Co., P. O. Box 351, Los Angeles 53,

California, Attn: J. B. Henderson

San Diegio Gas \& Electric Co., P. 0. Bolx 1831, San Diego 12, California 1

Attn: C. M. Laffoon 


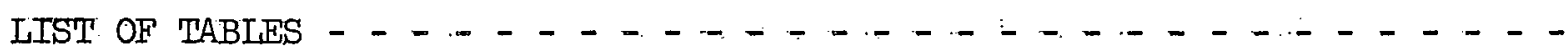

LIST OF FIGURES- _.

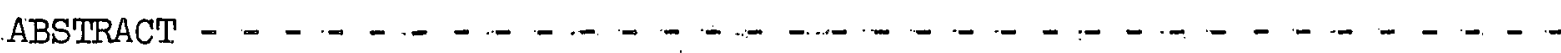

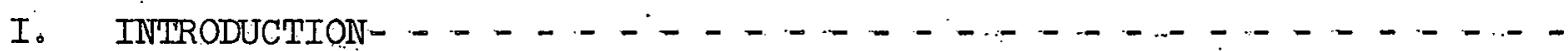

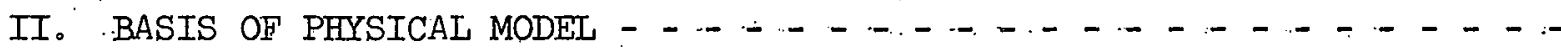

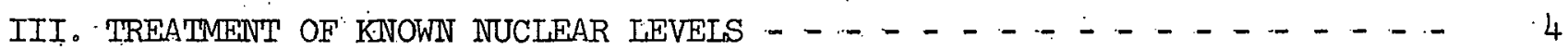

IV 。 INETASTIC SCATTERING PROBABITITTY IN STATISTICAL REGIONS - - - - - - 8

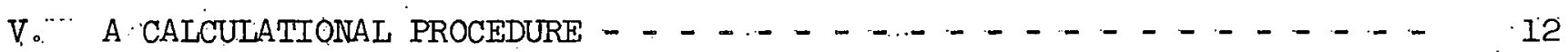

VI. CALNULLATION OF. INELASTIC SCATTHRING MATRIX FOR Fe $\mathrm{F}^{56} \ldots \ldots \ldots$

A. Experimental Data and Theoretical Formulas _. . . . . . . - 17

B. Numerical Results for Known. Nuclear Levels $\left(\mathrm{Fe}^{56}\right)-\ldots$

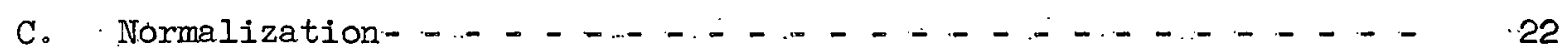

D. Calculations of Inelastic Scattering Probability $\left(\mathrm{Fe}^{56}\right)$

E $\left._{\text {incident }}<2.86 \mathrm{Mev}\right) \ldots \ldots 26$

E. Calculation of Inelastic Scattering Matrix ( $\left.\mathrm{Fe}^{56}\right) \ldots \ldots$

APPENDIX A - Inelastic Scattering Probabilities in the Statistical Region- A-1

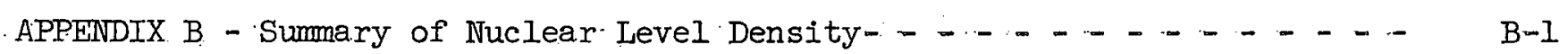

I. Models for Nuclear L'evel Density- _. . . . . . . . . B B

A. Fermi Degenerate Gas Model- _ _ - . - . . . - B-3

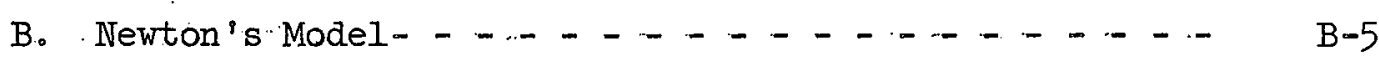

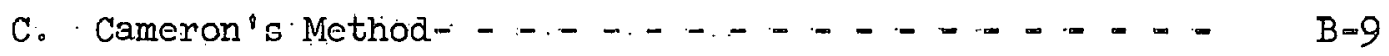

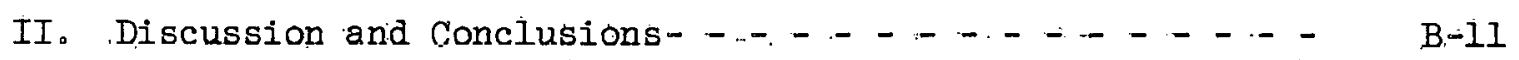

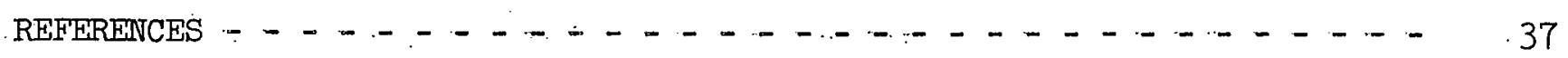




\section{LIST: OF ·TABLES}

Table No:

I

II

III

IV

$A-I$

$A \div I I$

$A-I I I$

B-I

$B-I I$
Titile

The Denominator Factors vs. Energy (Eqs. 21-27) for Fe

Calculated Inelastic Scattering Cross Section for

Various Levels of $\mathrm{Fe}^{56}$

Inelastic Scattering Probability Matrix for $\mathrm{Fe} \mathrm{s}^{56}$

Inelastic Scattering Probability Matrix for $\mathrm{Fe}^{56}$ in the Statistical Region:

Values of $t_{i}$ for MUFT Energy Groups

Normalized Inelastic Scattering Probabilities

Normalized Inelastic Scattering. Probabilities

Effective Proton Spin Parameters

Effect1ve Neutron Spin'Parameters
$A-2$

Páge No:

24

25

34

36

$A-8$

$A-9$

$B-7$

$B=8$ 
The Level Scheme of $\mathrm{Fe}^{56}$

2

3

4

5

$6(a)$

$6(b)$

$A=1$

$A=2$

$A=3$

$A=4$

A -5

B-1

B-2

Transmission Coefficients for $\mathrm{Fe}^{56}$ from Emmerich's Calculation

21

Comparison of Experimental Data and Calculated

Inelastic Scattering Cross Section for $\mathrm{Fe}^{56}$

Normalized $\sigma_{1}$, Normalized $\sigma_{0}, \sigma_{3}, \sigma_{4}$ and $\sigma_{5}$

Normalized $\sigma_{1}$, Normalized $\sigma_{2}, \sigma_{3}, \sigma_{4}$ and $\sigma_{5}$ versus Exit Neutron Liethargy

Calculated and Normalized $\sigma_{1}$ and $\sigma_{2}$ versus Permissible Values of Exit Neutron Lethargy for Incident Neutrons. in. MUFT Group 6

Calculated and Normalized $\sigma_{2}$ and $\sigma_{3}$ versus Permissible Values of Exit Neutron Lethargy for Incident Neutrons in MUFT Group 6

Calculated Inelastic Scattering Probabilities for Various MUFT Groups versus Nuclear Temperature

Calculated Inelastic Scattering Probabilities for Various MUFT Groups versus: Nuclear. Temperature

Calculated Inelastic Scattering Probabilities for Various MUFT Groups. versus Nuclear Temperature

Calculated Tnelastic Scattering Probabilities for Various MUFT Groups versus Nuclear Temperature

Calculated Inelastic Scattering Probabilities for Various MUFT Groups versus Nuclear Temperature

Level Spacing per Spin State versus Number. of Neutrons in Compound Nucleus (Harvey 1961) 
. A calculational procedure for obtaining the inelastic scattering probabilities, which are required for MUFT-4 - A Multigroup Fast Neutron Spectrum Code, is described. The relevant theoretical considerations are summarized. The formulation of Hauser and.Feshback is used when known nuclear levels can be excited. In all other cases, the statistical model is recommended. To elucidate the procedure an illustration is given for $\mathrm{Fe}^{56}$. In addition, general inelastic scattering probabilities are presented as a function of nuclear temperatures, and a critical discussion of the theoretical developments in the statistical model is given. 


\section{INTRODUCTION}

The treatment of neutron slowing-down is of significant importance in the design of thermal power reactors. There are two main mechanisms by which fission neutrons lose energy: (1) the elastic scattering and (2) the inelastic scattering processes. In this report, only the inelastic scattering of neutrons is discussed. By inelastic neutron scattering, it is implied that a neutron loses part of its energy by exciting the nucleus (in the ground state) to one of its excited levels. The probability of this process depends upon the specific nuclear levels of a nucleus. It turns out that all the nuclear levels, which can in principle be excited, are not known. Sometimes the relevant information like spins, parities and excitation energies may be known experimentally only for a few nuclear levels.. Consequently, a nuclear model has to be used in such calculations.

It is the purpose of this report to document a calculational procedure by which the inelastic-scattering-probability matrices, which appear in a multigroup code, ${ }^{1}$ could be calculated. It may be remarked at this stage that most of the formalism used appears already in the literature. For the sake of clarity and convenience, this report is divided into six sections. First, a treatment of the known nuclear levels is given in Section 3, followed by a discussion of the statistical region in Section 4. Section 5 contains an illustration, and ti:e numerical results are given for $\mathrm{Fe}^{56}$. A summary of the statistical model and the general numerical results are given in the Appendices $A$ and $B$ respectively. 


\section{BASIS OF PHYSICAL MODEL}

In 1936, N. Bohr presented a very simple idea for the mechanism of nuclear reactions - namely, the concept. of compound nucleus formation. Since then, this idea has been investigated thoroughly and surprisingly enough this concept, with some minor changes, is still considered valid. The formation of a compound nucleus implies that when a neutron (or any other particle, e.g. proton, $\alpha$ particle) is incident on a target nucleus, a compound system (consisting of the incident particle and the target nucleus) is formed. The decay time of such a compound system" is generally large compared to the nuclear time scale. Because of this reason, an incident.neutron loses its identity in the compound system. Thus, in the compound system formation, it is explicitly assumed that the properties of this compound system are independent of how it was formed. For example, whether a compound system with certain quantum numbers (total angular momentum, parity and energy) is formed by an incident neutron or a proton, the decay of this system is independent of the incident particle. As is to be expected, this compound system prefers to decay giving off neutrons compared to protons, because of the strong coulomb effects in the case of a charged particle.

In many cases, a large number of compound states can be excited because of the following two reasons:

1) the nuclear level spacing may be less than the width of these levels and,

2) the spread in the incident energy. of neutrons is larger than the level spacing.

If, in addition, numerous nuclear levels of a residual nucleus can be excited, we can treat the decay of the compound system in a statistical fashion. A strict test of the formation of a compound system is the prediction of isotropic angular distributions of emergent particles around $\theta=\pi / 2$. 
(This means terms with only. 2L appear in the Legendere polynomial). This isotropy has been observed in more than $90 \%$ of the cases.

At this stage $;$ we point out that the inelastic scattering calculations become more difficult:when the statistical assumption of compound-system decay. is not valid. For example, when only a few nuclear levels can be excited (because of the incident neutron energy) one has to use a different (physical), nuclear model. The ground work of such analyses was first proposed ${ }^{2}$ by Hauser and Feshbach in 1952. In this nuclear model, an average nuclear potential is assumed and then the wave-equation is solved so that "transmission coefficients" can be calculated. 3,4 It became obvious that this average nuclear potential should have at least two parts, namely a real part and an imaginary part. In most calculations, it has been assumed that this imaginary, part of the nurlear potential is related to the real part. Further refinements - like a diffusive boundary, of the nuclear potential were incorporated later on. A detailed account of these new developments appear in the review articles ${ }^{5}$ by Bodansky ${ }^{6}$ (1962), Feshbach ${ }^{7}$ (1960) and the original reports of Emmerich ${ }^{4}$ (1962).

Another new process has been discovered recently. It is called the "direct interaction" or "nuclear stripping." In this process, an incident particle does not spend so much time in the compound system and consequently retains most of its identity. This results in a strong peak in the angular distributions of emergent particles. However, it.turns out that there are rclatively few cases (less than $10 \%$ ). So far this process has been ignored. in reactor calculations. In our opinion, this cannot lead to any significant effects in a typical reactor calculations. A summary 8,9 of the physical model for nuclear stripping is given by Bannerjee (1960) and Levinson (1960). 
In this report, by known nuclear levels, we will 1mply that the spin excitation energy and parity of the levels have been deduced from experimental data. In this section, we consider the case when the incident neutron energy is such that only known levels can be exc1ted. We will define $\sigma\left(1 \longrightarrow 1^{\prime}, E \rightarrow E^{\prime}\right)$ as the cross section for the inelastic scattering of incident neutrons with energy $E$ from a target nucleus with ground state spin 1 and parity $P(1)$ with the target nucleus being left in an excitated state with spin $1^{\prime}$, parity $P_{\left(1^{\prime}\right)}$ and excitation energy $E-E^{\prime}$. The energies of the incident and the inelastically scattered neutron, namely $\mathbf{E}$ and $\mathrm{E}^{\prime}$, are in Mev and in the center of mass system. Following Hauser and Feshbach, one gets:

$$
\sigma\left(1 \rightarrow 1^{\prime}, E \rightarrow E^{\prime}\right)=\frac{\pi}{2(21+1)} \hbar^{2} \sum_{b=0}^{\infty} T_{\ell^{\prime}}(E) \sum_{J=1-\left(b+\frac{1}{2}\right)}^{1+\ell+\frac{1}{2}} \epsilon_{1, \ell^{J}}^{(2 J+1) N^{J}}
$$

where:

$$
N^{J}=\frac{\sum_{\ell^{\prime}=0}^{\infty} \epsilon_{1^{\prime}, \ell^{\prime}}^{J} T_{\ell^{\prime}\left(E^{\prime}\right)}\left|(-1)^{P}(1)+\ell+(-1)^{P}\left(1^{\prime}\right)+\ell^{\prime}\right|}{\sum_{q} \sum_{\ell^{\prime \prime}=0}^{\infty} \epsilon_{1(q), \ell^{\prime \prime}}^{J} T_{\ell^{\prime \prime}}\left(\mathrm{B}^{\mathrm{J}} \mathrm{E}_{\mathrm{q}}\right)\left|(-1)^{\mathrm{P}}(1)+\ell+(-1)^{\mathrm{P}}(\mathrm{q})^{+\ell^{\prime \prime}}\right|}
$$

with the following definitions:

$$
\begin{aligned}
* & =\text { de Broglie wavelength of the incident neutron divided by } 2 \pi \\
1 & =\text { spin of the ground state of the finget nucleus } \\
P_{(1)} & =\text { pailty of the givuid slale of lie largel iucleus }
\end{aligned}
$$


$i^{*}=$ spin of the excited level of the nucleus

$P_{(1 !)}=$ parity, of the excited level of the nucleus

$q=$ this index implies appropriate quantum numbers of all the levels up. to $\mathrm{E}_{\mathrm{q}} \leq \mathrm{E}_{\text {。 }}$

$E:=$ energy (in Mev), of the incident.neutron in C-system.

$E^{\prime}=$. energy (in Mev) of the inelastically: scattered neutron in D-system.

$E_{i}=E-E^{r}=$ energy (in Mev) of the excited level

$\ell=$ orbital angular momentum of the incident neutron

$\ell^{\prime}=$ orbital angular momentum of the inelastically. scattered neutron

$J \rightleftharpoons$ total angular momentum of the compound system.

$\mathrm{T}_{\ell}(\mathrm{E})=$ transmission coefficient corresponding to a particular orbital angular momentum $l$ and energy (in Mev) of a neutron. $E$ is in C-system.

$\sigma\left(i \rightarrow i^{\prime}, E \rightarrow E^{\prime}\right):=$ cross section in $\ddot{c m}^{2}$ for neutrons with energy $E$ in Mev inelastically, scattering from a target.nucleuss with spin $i$ and parity $P(i)$. leaving the target nucleus in an excited state with spin $i^{\prime}$, parity $P\left(i^{\prime}\right)$ and excitation

$\epsilon_{1, l}^{J}=\left\{\begin{array}{l}2, \text { if conditions } I \text { and } I I \text { are satisfied } \\ 1, \text { if. either condition I or condition II is satisfied } \\ 0, \text { if neither condition I. or II is satisfied }\end{array}\right.$

$$
\begin{aligned}
& \text { Condition I J-l } \leq i+\frac{1}{2} \leq J+\ell \\
& \text { Condition II J-l } \leq i-\frac{1}{2} \leq J+\ell
\end{aligned}
$$

It may be noted that the permissable values of J are obtalned by. vector adalthon of $\vec{I}$ and ( $\vec{b} \vec{b})$, where $\overrightarrow{\vec{l}}$ and $\vec{f}$ are the orbital angular momentum and spin of the incident neutron. 
The calculation of the transmission coefficients; $T_{l}(E)$, involves an assumption of an average nuclear potential with real and imaginary parts. Emmerich ${ }^{4}$ has tabulated the transmission coefficients for a wide range of parameters. Emmerich uses the following potential:

$$
\begin{aligned}
& V(r)=-V_{c} \text { for } r<R_{c} \\
& V(r)=-V_{c}\left(1-i \lambda \frac{d}{d x}\right) \cdot\left[e^{x}-x\right]^{-1} \text { for } r>R_{c}
\end{aligned}
$$

where

$$
x=\frac{r-R_{c}}{\tau}
$$

The diffusive edge is determined by the value of $\tau$. Note that the imaginary part of the nuclear potential was taken to zero in the core and $\lambda$ times the derivative of the real part with respect to $x$ in the surface region. : The mean radius, $\bar{R}$,. was defined as follows:

$$
\overline{\mathrm{R}}=\int_{0}^{\infty} \frac{\mathrm{V}(r)}{\mathrm{V}_{\mathrm{c}}} \cdot \mathrm{dr}
$$

The $e_{i}$ above equation leads to $\overline{\mathrm{R}}=-\mathrm{R}_{\mathrm{c}}+1.359 \mathrm{~T}$

Then, we have four parameters to describe the potential, namely. $\bar{R}, \tau, V_{c}$ and $\lambda$. By a comparison : with experimental data, it was found for an isotope of mass number A (See for example, Emmerich-reference 4)

$$
\bar{R}=\left(1.25 A^{\frac{1}{3}}+0.5\right) \cdot 10^{-13} \mathrm{~cm}
$$

Finmerich has tabulated the transmission coefficient up to $l=8$ for the following cases for $\bar{R}$ from 4.0 to 8.2 in intervals of 0.3 (units of $10^{-13} \mathrm{~cm}$ )

\begin{tabular}{|c|l|l|}
\hline$v_{c}$ & $\lambda$ & E(in Mev $)$ \\
\hline 44 & 0.34 & $0.1,0.4,0.7,1.0$ \\
43 & 0.37 & $1.5,2.0,2.5,3.0$ \\
42 & 0.40 & $4.0,5.0,6.0$ \\
41 & 0.43 & $7.0,8.0,9.0,10.0$ \\
40 & 0.46 & $12.0,14.0$ \\
\hline
\end{tabular}


It should be noted that it has been assumed that the transmission coefficients do not depend on the total angular momentum of the compound system.

At this stage, we give detailed expressions of Eq. (1) for one case of interest. The summations in Eq. (1) and Eq. (2) are rather intricate, and some care must be used in obtaining appropriate results for the case of interest: :

If we have the lst excited state $(2+)$ and the ground state $(0+)$ and the neutron energy is such that only. lst.excited state can be excited, we have

$$
\begin{aligned}
& \sigma\left(0^{+} \rightarrow 2^{+}\right)=\frac{\pi-x^{2}}{2} \cdot\left[T_{0}(E) \frac{2 T_{2}\left(E^{t}\right)}{2 T_{2}\left(E^{t}\right)+T_{0}(E)}\right. \\
& +T_{1}(E)\left\{\frac{2\left[T_{1}\left(E^{\prime}\right)+T_{3}\left(E^{\prime}\right)\right]}{T_{1}\left(E^{\prime}\right)+T_{3}\left(E^{\prime}\right)+T_{1}(E)}+\frac{4\left[2 T_{1}\left(E^{\prime}\right)+2 T_{3}\left(E^{\prime}\right)\right]}{2 T_{1}\left(E^{\prime}\right)+2 T_{3}\left(E^{\prime}\right)+T_{1}(E)}\right\} \\
& +T_{2}(E)\left\{\frac{4\left[T_{0}\left(E^{\prime}\right)+2 T_{2}\left(E^{\prime}\right)\right]}{T_{0}\left(E^{\prime}\right)+2 T_{2}\left(E^{\prime}\right)+T_{2}(E)}+\frac{6\left[T_{0}\left(E^{3}\right)+2 T_{2}\left(E^{8}\right)\right]}{T_{0}\left(E^{8}\right)+2 T_{2}\left(E^{\prime}\right)+T_{2}(E)}\right\} \\
& \left.+T_{3}(E)\left\{\frac{6\left[2 T_{1}\left(E^{\prime}\right)+2 T_{3}\left(E^{\prime}\right)\right]}{2 T_{1}\left(E^{\prime}\right)+2 T_{3}\left(E^{\prime}\right)+T_{3}(E)}+\frac{8\left[T_{1}\left(E^{\prime}\right)+2 T_{3}\left(E^{\prime}\right)\right]}{T_{1}\left(E^{\prime}\right)+2 T_{3}\left(E^{\prime}\right)+T_{3}(E)}\right\}\right]
\end{aligned}
$$

:Here $E^{\prime}=E_{1}-E_{1}$, where $E$ and $E_{1}$ are the incident neutrons energy and the excitation energy, of $2^{+}$level respectively. All these energies are in the center of mass system. In heavy materials, where recoil energy of the residual nucleus is very small, the energies in C-system are essentially the same as in the laboratory. frame. It should be noted that energy-groups in MUFT correspond to the laboratory frame of reference.

$$
E_{c}=\left(\frac{A}{A+1}\right) E_{L}
$$

In $\mathrm{Eq} \cdot(5)$

$$
\pi^{2}=\frac{.20728}{E(\text { in Mev })} \cdot 10^{-24} \mathrm{~cm}^{2}
$$

where $\mathrm{E}$ is the neutron incident energy. in C-system. 


\section{INELASTTC SCATTERING PROBABILITY IN STATISTICAL REGIONS}

By a statistical region, we imply the energy region of excited nuclear levels whenever these nuclear levels are so numerous (and close to each other) that a statistical treatment of a compound system can be made. At the very autset, it may be noted that very. little experimental information as regards spins and parities of the nuclear levels is available beyond 3 mev or so. Consequently, some sort of nuclear model must be used for a description of the nuclear levels above 3 mev. Usually. the particular choice of the dependence of level density of excitation energies is dictated by experimental measurements of either the $\gamma$-ray measurements or energy distribution of inelastically. scattered neutrons. The measurements of $\gamma$-ray. intensities give information about inelastic scattering because one can "identify" the $\gamma$-ray which reeults from the transition between the excited nuclear states to the ground state. The statistical approach of the decay of a compound system (consisting, of the incident neutron and the target nucleus), is sometimes compared to the evaporation of a Iiquid arop, where evaporation is governed by purely statistical consideration. Thus, in analogy with thermodynamics, it has become customary to define a nuclear temperature. These considerations give us an expression for the relative intensity. of.a particle from a statistical decay of a compound system.6

$$
I_{v}\left(E, E^{*}\right) d E=\frac{\left(2 S_{\nu}+1\right) \cdot E \sigma_{\nu}(E, U) \rho_{\nu}(u) M_{\nu} d E}{\pi^{2} \hbar^{3} \rho_{c}\left(E^{*}\right)}
$$

where:

$$
\begin{aligned}
I_{v}\left(\mathrm{E}, \mathrm{E}^{*} \cdot\right) \mathrm{dE}= & \text { relative yield of particle } v \text { with energy } \mathrm{E} \text { when a } \\
& \text { compound system with total energy } \mathrm{E}^{*} \text {.decays. } \\
\mathrm{U}= & \mathrm{E}^{*}-\mathrm{E}=\text { Excitation energy, of a level } \\
\mathrm{M}_{v}= & \text { mass of the particle } v \\
\mathrm{~S}_{v}= & \text { spin of the particle } v \\
\rho_{v}(U)= & \text { nuclear level density at excitation energy of } U_{0}
\end{aligned}
$$




$$
\begin{aligned}
\pi= & \text { Planck's constant divided by } 2 \pi \\
\sigma_{v}(E ; U)= & \text {. } \\
& \text { pross section for formation of a compound system when a } \\
v= & \text { symbol, designating a particle e.g. a neutron, a proton, } \\
& \alpha \text { particle etc. etc. }
\end{aligned}
$$

In a reactor calculation; we are concerned mainly.. with neutron fluxes. Thus, we get for neutrons

$$
I(E),=C \cdot E \sigma_{c}(E, U) \cdot o(U) \cdot d E
$$

Here, we have absorbed the various constant factors in the constant $C_{\text {a }}$ Let us wake another assumption $i_{\circ} e_{0} \sigma_{c}(E, U)$ has very weak energy. dependence, and therefore can also:be absorbed in the constant $C$. We wish to point out this assumption appears to be valid. However, this validity is based upon certain other assumptions. This is because it is extremely. difficult (if not impossible) to measure a capture cross section for a certain incident neutron energy on an "excited" nuclear level. This has led to the assumption that perhaps this cross section can be given by the corresponding:cross section for the ground state. For the ground state, the reaction cross section, $\sigma_{r}$, is given by the following:

$$
\sigma_{r}(E)=\pi \pi^{2} \sum_{i=0}^{\infty}(2 \ell+1) T_{h}(E)
$$

: where $\mathbb{T}_{\ell}(E)$, is the transmission coefficient. for neutron energy $E$ and orbital angular.momentum $\ell$, and $\lambda$ is neutron wave length divided by $2 \pi$. By explicit calculations, it can be seen that $\sigma_{r}(E)$, does not vary significantly, with energy above $4 \mathrm{mev}$ or so. In this statistical region (neutrons above 3 mev

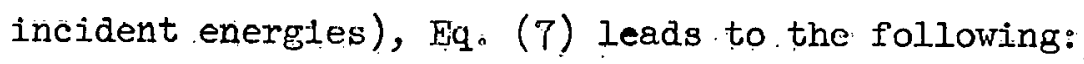

$$
I\left(E_{g} E_{0}\right) d E=C E \rho\left(E_{0}-E\right) d E
$$


In Eq. (9), we have shown the functional dependence of relative neutron flux with energy $E$ for incident, neutrons of energy $E_{0^{\circ}}$

In one of the simplest. forms, which has been used widely, we have

$$
\rho\left(E_{0}-E\right)=C_{1} e^{\left(E_{0}-E\right) / T}
$$

where $\mathrm{C}_{1}$ is a normalizing constant. The nuclear temperature, $\mathrm{T}$, is considered as a function of the incident neutron energy $\mathrm{E}_{\mathrm{O}^{\circ}}$ This particular form of the nuclear level density. implies that the energy-dependence is contained in the exponential term. Though there are no theoretical arguments to support.this form; yet:most of the experimental results have been used in arriving at the values of nuclear temperature. Using Eqs. (9), and (10), we get the probability of a neutron (with lethargy $u$ ) to be inelastically scattered with final lethargy value $u^{\prime}$ 。

$$
P\left(u \rightarrow u^{\prime}\right)=\frac{I}{T^{2}}\left[E^{\prime 2} \mathrm{e}^{-E^{\prime} / T}\right]: d u^{\prime}
$$

The nuclear temperature, T, can be expressed as a function of the incident neutron energy.

$$
T=a E^{\frac{1}{2}}
$$

where the parameter a is determined from experimental data. It should be noted that this parameter, a, is different for each isotope In MUFT, the energy-groups are defined frôli a cextain energy $E_{i}$ to $E_{1=1}$. For the calcula.tion of inelastic scattering probabilities from a group $i$ to other groups, we need an "average" nuclear temperature for the groups; designated by. i. This should be done by the following equation.

$$
T_{i}=\frac{2 a}{\Delta u_{1}}\left[E_{i}^{\frac{1}{2}}-E_{i-1}^{\frac{1}{2}}\right]
$$

:where $E_{i}$ is the larger energy value in group $i$ and $\Delta_{i}$ is the lethargy interval of group $i$. The nuclear temperature is a positive definite number. 
Thus, we get the probability of inelastic neutron scattering from group $i$ to energy group $j$, where $j>i$.

$$
\left.P_{i j}=\left(\frac{E}{T_{i}-1}+1\right) e^{-E_{j-1} / T_{i}} \cdot \frac{E_{j}}{T_{i}}+1\right) e^{-E_{j} / T_{i}}
$$

Again, it must be noted that $E_{j-I}<E_{j}$ and this leads to the obvious statement that $P_{i j}>0$ for $j>i$.

At this stage, it is to be noted that several other forms of the nuclear level density have been proposed. For example,

$$
\rho(U) \propto U^{-n} e^{2 \sqrt{b U}}
$$

where $n$ has been given values of $5 / 4,2$ and 0 . For the case $n=0$, (as in Eq. 10) it is tacitly assumed that only the exponential term governs the dependence of the nuclear level density. on the excitation energy. . In this formulation the temperature, $\mathrm{T}$, is related to $\mathrm{n}$ and $\mathrm{b}$ by

$$
\frac{1}{T}=\left(\frac{b}{U}\right)^{\frac{1}{2}}-\frac{n}{U}
$$

It may be noted, here, that only when $n=0$ and $b$ is constant we get the relation

$$
T=b U^{\frac{1}{2}}
$$

Another interesting point is that the value of the level density at a certain energy is not the same for odd-odd nuclei and even-odd nuclei. In fact, we have the relationship approximation:

$$
\begin{aligned}
& : 0.5 C_{1} \text { (odd-odd) }=C_{1} \text { (odd-even, even-odd) } \\
& C_{1} \text { is a parameter in Eq. (10) }
\end{aligned}
$$

A summary of the expressions for the nuclear level density is given in Appendix $B$. 
In this section, a calculational procedure for obtaining an inelastic scattering matrix for a certain isotope is summarized. For more detailed information than given below, original papers and the recent review article should be consulted.

Step 1: Make a literature search on the experimental data for an isotope. The following sources should be consulted first:

(a) Nuclear Data Sheets

(b) Reviews of Modern Physics, 30, No. 2 Part II (1958)

(c) BNL - 325 (and supplements)

The above references should, at least, establish the nuclear level scheme. It may be of a great help if the same information is requested from:

Sigma Center, Brookhaven National Laboratory. Perhaps, the Sigma Center can also supply the references to other later experimental data. In any case, some recent Phýsical Reviews and Nuclear Science Abstracts should also be searched. The following information must be compiled:

(1) Nuclear level structure of an isotope,

(2) Experimental $\sigma_{n, n}$, cross section for the measured levels

(3) The nuclear temperature, T, at a certain energy.

If some of thece data are not avajlahle, it will be necessary to use similar information concerning a neighboring isotope.

Step 2: Obtain the appropriate transmission coefficients. This can be done by first calculating

$$
\bar{R}=\left(1.25 \mathrm{~A}^{\frac{1}{3}}+0.5\right) 10^{-13} \mathrm{~cm}
$$

and interpolating between the values from Emmerich's calculations (Westinghouse Research Report 62-118-540-P1, 1962). Alternatively, the values 
of transmission coefficients can be obtained from Los Alamos Scientific Report LA-2099 (April. 1957), MIT - INS No. 73 (February 1960) or by running a code. It may be noted that these transmission coefficients depend very: much on the nature of the average nuclear potentials used. It turns out that:most of these calculations use slightly different.models and also the results are presented differently.

It.is suggested that the transmission coefficients, calculated by using any of the above methods, be plotted versus neutron energy for the isotope of interest.

Step 3: If the nuclear temperature is not:known experimentally (as in Step 1), one can use a value either from an empirical fit of Newton ${ }^{13,14}$ (Canadian J. of Physics 34 , 804 (1956), or a value may be calculated by using the more recent curves of $\mathrm{b}$.in-Eq. (16). (Ericson,. Proceeding of the International Conference on Nuclear Structure, University of Toronto - Press (1960).

Step 4: $:$ Consider the contributions of all known nuclear levels. (This means that one knows the spin, the parity and energy. of excitation of the relevant nuclear levels。) Using Eq. (1) and the curves (Transmission Coefficients vs Energy), calculate $\sigma_{\text {in }}$ for the first excited level and denote it as. $\sigma_{\text {in }}(1)$. It may be noted that the energy factor in Eq. (I): refers to the center of mass. system. We suggest that all calculatioris be done in this c-sysleï, and latcr on, if need be, nentron energies be converted to the laboratory system. It may "be recalled that since $E_{c}=\left(\frac{A}{A+1}\right) E_{L}$ we can ignore the distinction between $E_{L}$ and $E_{c}$ : when $A$ is large $(A>50)$.

Plot $\sigma_{\text {in }}(1)$, the calculated inelastic cross section for the first -excited level, versus neutron lethargy. Similarly plot: $\sigma_{\text {in }}(j)$,vs-neutron lethargy for all known nuclear levels $i . e . j=2,3,4 \ldots \ldots$ lethargy, $u_{j}=\log \frac{10}{\mathrm{E}(\text { in Mev })}$. 
Step 5: Since MUFT does not allow inelastic scattering within a group, this contribution has to be properly considered in the values of $\sigma_{\text {in }}$ The appropriate method is to calculate

$$
f_{i}=\frac{\sum_{i, j}^{\prime} \sigma_{i, j}}{\sum_{i, j}}
$$

where the prime over the summation denotes $j \neq i$. These fractions, $f_{i}$; should be calculated for all the relevant energy groups. The enteries $\sigma_{\text {in }}^{j}$ (for an energy-group designated by $j$ ) should be calculated as- follows:

$$
\dot{\sigma}_{\text {in }}^{j}(M U F T)=f_{j} \sigma_{i n}^{j}(\operatorname{expt})
$$

Note that $\left(I-f_{j}\right): \sigma_{i n}^{j}($ expt。 $)$ should be put in MUFT as symmetric scattering。

Step 6: From Step 4, we have $\sigma_{\text {in }}$ (for various known levels) versus neutron energy (generally up to $3 \mathrm{Mev}$ or so.) . Now it may turn out that these respective cross sections do not agree with experimental information. The experimental cross. sections may be available only at a certain energy. In such a case, normalize the calculated $\sigma_{\text {in }}$ to the experimental value.

Step 7: Calculate the inelastic scatterlng probabilities for the known nuclear levels as follows:

$$
p_{1 J}=\frac{\sigma_{i j}}{\sum_{i, j}^{i} \sigma_{i, j}}
$$

$\sigma_{i, j}$ is the lethargy averaged cross sections (calculated from the theoretical curves normalized to experimental data), so that a neutron incident in group 1 can be ecattered (inelastically) into energy group jo It :should be noted that this step is not difficult, because for a certain incident energy (in group i) only discrete values of exit.neutron energies are possible. Therefore, sometimes group $j$ may not be completely subscribed by the neutrons in group $i$. 
Since MUFT energy-groups are defined. in a simple fashion in units of lethargy, these calculations can be done conveniently when $\sigma_{\text {in }}$ (for all the nuclear levels) is plotted versus lethargy.

Step 8: When the incident neutron energy is greater than the last resolved level (spin \& parity known), we cannot continue the above procedure because some levels, where spins and parities are not known, can now be excited. This results in the usual difficulty because the appropriate transmission coefficients (in the denominator of Eq. (2)) cannot be calculated. The procedure recommended is that above 3 (or 4) Mev energies, one should use the statistical model. We will remark on this interference between the resolved and the unresolved nuclear level regions in Part $C$ of the report. However, we suggest that this interference contribution be ignored at least for the immediate future.

Step 9: When all the inelastic scattering probabilities of the known levels have been computed, use the appropriate nuclear temperature from step 1 in the equation.

$$
T=a E^{\frac{1}{2}}
$$

to determine the parameter a. The nuclear temperature for each energy group should then be computed from the following:

$$
T_{I}=\frac{2 a}{\Delta u_{1}}\left[E_{i}^{\frac{1}{2}}-\frac{1}{\frac{1}{2}} E_{i-1}\right]
$$

where $E_{i}$ and $E_{i-1}$ are the upper and the lower limits of energy specifying the group $i . \Delta u_{i}$ is the width of group $i$.

Step 10: With the nuclear temperatures calculated for all groups (above $3 \mathrm{Mev}$ or so), one calculates the irelastic scattering probabilities from the following equation:

$$
P_{i j}=\left(\frac{E_{j-1}}{T_{i}}+1\right) e^{-E_{j-1} / T_{1}}-\left(\frac{E_{j}}{T_{i}}+1\right) e^{-E_{j} / T_{i}}
$$


Step 11: Any finite contribution of inelastic probability to groups beyond energy-group 25. of MUFT can either:be lumped in group 25. or can be distributed uniformly, over all the energy groups .

Step 12: Summations of probabilities should be normalized to 1.000. This is a strict condition in MUFT library。

$$
\sum_{j} p_{i j}=1.0000
$$




\section{CALCULATION OF INELASTIC SCATTERING MATRIX FOR Fe}

In our calculations, we make the assumption that a statistical description is valid above $2.86 \mathrm{Mev}$. Furthermore, since $\mathrm{E}_{\mathrm{C}}=\left(\frac{56}{56+1}\right) \mathrm{E}_{\mathrm{L}}$ we ignore the distinction between $E_{C}$ (energy in $C$-System) and $E_{L}$ (energy in the laboratory frame of reference). As it.can be easily seen in the calculations presented here, this approximation can be easily dispensed with。

A Experimental Data and Theoretical Formulas

The level scheme of $\mathrm{Fe}^{56}$ was taken ${ }^{3}$ as in Fig. 1.

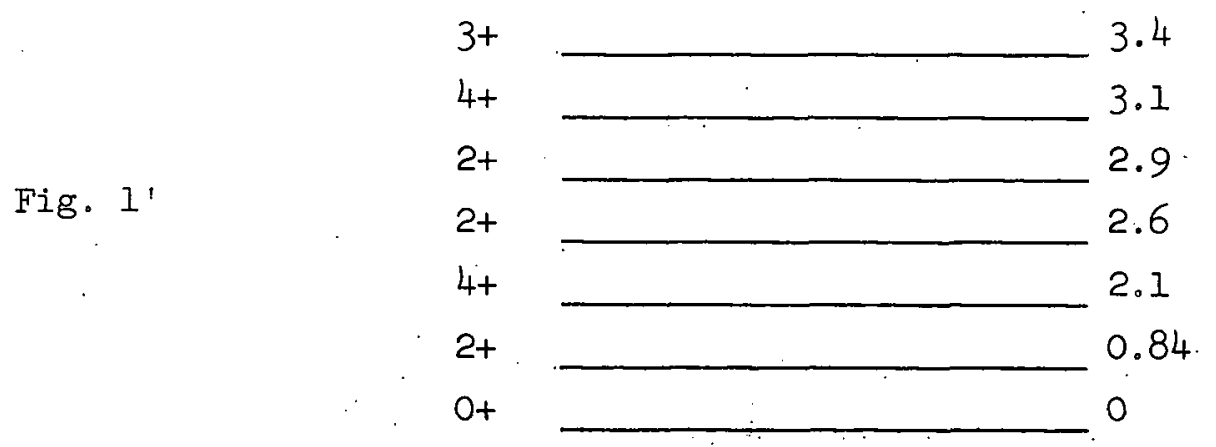

In this scheme, the ground state has spin zero and even parity. The first level (2+), the second level (4t), the third level (2+), the fourth level (2+), the fifth level $(4+)$ and the sixth level (3+) are respectively at the following energies (with respect to the ground state): $0.84 \mathrm{Mev}$, 2.1. Mev, 2.6 Mev, $2.9 \mathrm{Mev}, 3.1 \mathrm{Mev}$ and $3.4 \mathrm{Mev}$. It may be noted that the spin of the nuclear level at $3.1 \mathrm{Mev}$ is not completely established. In fact, it has been suggested ${ }^{15}$ that this spin may be 2 instead of 4. Thomas ${ }^{16}$ and Bodansky ${ }^{6}$ have summarized the recent experimental information. It has been found ${ }^{6}$ that the value of nuclear temperature $T=0.95$ for incident energy of $7 \mathrm{Mev}$. It should be noted that this nuclear temperature for $F e^{56}$ may be in error. There are conflicting experimental data in the literature. In fact, at the present time, the nuclear temperature (for $\mathrm{Fe}^{56}$ ) calculated from $\left(n, n^{\prime}\right)$ reactions is not consistent with the corresponding temperature 
for $\left(\mathrm{p}, \mathrm{p}^{\prime}\right)$ and $(\mathrm{p}, \alpha)$ reactions. The reasons for this discrepancy are not quite understood. See reference (17) for further discussion of this point.

In Fig. 1, we can have the following types of transitions:

$$
\mathrm{O}+\rightarrow 2+, \mathrm{O}+\rightarrow 4+\text {, and } \mathrm{O}+\longrightarrow 3+\mathrm{O}
$$

We give below the inelastic cross sections for these combinations up to $\ell$ and $l^{\prime}$ equal ${ }^{*}$ to $3 . \ell$ and $l^{\prime}$ are the orbital angular momentum of the incident and the (inelastically). scattered neutron respectively.

From Eq: (1), one obtains:

$$
\begin{aligned}
& \sigma(0+\rightarrow 2+)=\frac{\pi x^{2}}{2}\left[T_{0}(E) \cdot \frac{4}{A} T_{2}\left(E^{2}\right)\right. \\
& +T_{1}(E)\left\{\frac{2}{B}\left[T_{1}\left(E^{\prime}\right)+T_{3}\left(E^{\prime}\right)\right]+\frac{8}{C}\left[2 T_{1}\left(E^{\prime}\right)+2 T_{3}\left(E^{\prime}\right)\right]\right\} \\
& \text { it } T_{2}(E)\left\{\frac{4}{D} \cdot\left[T_{0}\left(E^{\prime}\right)+2 T_{2}\left(E^{\prime}\right)\right]+\frac{6}{F}\left[T_{0}\left(E^{\prime}\right)+2 T_{2}\left(E^{\prime}\right)\right]\right\} \\
& \left.+T_{3}(E)\left\{\frac{12}{H} \cdot\left[T_{1}\left(E^{\prime}\right)+T_{3}\left(E^{\prime}\right)\right]+\frac{8}{K}\left[T_{1}\left(E^{\prime}\right)+2 T_{3}\left(E^{\prime}\right)\right]\right\}\right] \\
& \sigma(0+\rightarrow 3+)=\frac{\pi \star^{2}}{2}\left[T_{0}(E) \cdot \frac{2}{A}: T_{2}\left(E^{\prime}\right)\right. \\
& +T_{1}(E)\left\{\frac{4}{B} T_{3}\left(E^{\prime}\right)+\frac{4}{C}\left[T_{1}\left(E^{\prime}\right)+2 T_{3}\left(E^{\prime}\right)\right]\right\} \\
& +T_{2}(E)\left\{\frac{8}{D} T_{2}\left(E^{\prime}\right)+\frac{6}{F^{\prime}}\left[T_{0}\left(E^{\prime}\right)+2 T_{2}\left(E^{\prime}\right)\right]\right\} \\
& +T_{3}(E)\left\{\frac{12}{H}\left[T_{1}\left(E^{8}\right)+T_{3}\left(E^{\prime}\right)\right]+\frac{16}{K}\left[T_{1}(E !)+T_{3}(E)\right]\right\}
\end{aligned}
$$

*The values of the relevant transmission coefficients for $\ell \cdot>3$ are negligibly small for $\mathrm{E} \leq 2.86 \mathrm{Mev}$. See for example Fig. (2). 


$$
\begin{aligned}
\sigma(0+\rightarrow 4+) & =\frac{\pi \pi^{2}}{2}\left[\mathrm{~T}_{1}(E)\left[\frac{2}{\mathrm{~B}} \mathrm{~T}_{3}\left(\mathrm{E}^{\prime}\right)+\frac{8}{\mathrm{C}} \mathrm{T}_{3}\left(\mathrm{E}^{\prime}\right)\right]\right. \\
& +\mathrm{T}_{2}(\mathrm{E})\left[\frac{4}{\mathrm{D}} \cdot \mathrm{T}_{2}\left(\mathrm{E}^{\prime}\right)+\frac{12}{\mathrm{~F}} \mathrm{~T}_{2}\left(\mathrm{E}^{\prime}\right)\right] \\
& \left.+\mathrm{T}_{2}(\mathrm{E})\left\{\frac{6}{\mathrm{H}}\left[\mathrm{T}_{1}\left(\mathrm{E}^{\prime}\right)+2 \mathrm{~T}_{3}\left(\mathrm{E}^{\prime}\right)\right]+\frac{16}{\mathrm{~K}}\left[\mathrm{~T}_{1}\left(\mathrm{E}^{\prime}\right)+\mathrm{T}_{3}\left(E^{\prime}\right)\right]\right\}\right]
\end{aligned}
$$

where: ${ }^{*}$

$$
\begin{aligned}
A & =T_{0}(E)+2 T_{2}\left(E_{1}\right)+2 T_{2}\left(E_{3}\right)+2 T_{2}\left(E_{4}\right)+T_{2}\left(E_{6}\right) \\
B & =T_{1}(E)+T_{1}\left(E_{1}\right)+T_{3}\left(E_{1}\right)+T_{3}\left(E_{2}\right)+T_{1}\left(E_{3}\right)+T_{3}\left(E_{3}\right)+T_{1}\left(E_{4}\right) \\
& +T_{2}\left(E_{4}\right)+T_{3}\left(E_{5}\right)+2 T_{3}\left(E_{6}\right) \\
C & =T_{1}(E)+2 T_{1}\left(E_{1}\right)+2 T_{3}\left(E_{1}\right)+2 T_{3}\left(E_{2}\right)+2 T_{1}\left(E_{3}\right)+2 T_{3}\left(E_{3}\right) \\
& +2 T_{1}\left(E_{4}\right)+2 T_{3}\left(E_{4}\right)+2 T_{3}\left(E_{5}\right)+T_{1}\left(E_{6}\right)+2 T_{3}\left(E_{6}\right) \\
D & =T_{2}\left(E_{1}\right)+T_{0}\left(E_{1}\right)+2 T_{2}\left(E_{1}\right)+T_{2}\left(E_{2}\right)+T_{0}\left(E_{3}\right)+2 T_{2}\left(E_{3}\right) \\
& +T_{0}\left(E_{4}\right)+2 T_{2}\left(E_{4}\right)+T_{2}\left(E_{5}\right)+2 T_{2}\left(E_{6}\right) \\
& =T_{2}\left(E_{)}\right)+T_{0}\left(E_{1}\right)+2 T_{2}\left(E_{2}\right)+T_{0}\left(E_{3}\right)+2 T_{2}\left(E_{3}\right)+T_{0}\left(E_{4}\right) \\
& +2 T_{2}\left(E_{4}\right)+2 T_{2}\left(E_{5}\right)+T_{0}\left(E_{6}\right)+2 T_{2}\left(E_{6}\right) \\
& =I_{3}\left(H^{\prime}\right)+2 T_{1}\left(E_{1}\right)+2 T_{3}\left(E_{1}\right)+T_{1}\left(E_{2}\right)+2 T_{3}\left(E_{2}\right)+2 T_{1}\left(E_{3}\right) \\
& +2 T_{3}\left(E_{3}\right)+2 T_{1}\left(E_{4}\right)+2 T_{3}\left(E_{4}\right)+T_{1}\left(E_{5}\right)+2 T_{3}\left(E_{5}\right)+2 T_{1}\left(E_{6}\right)+2 T_{3}\left(E_{6}\right)
\end{aligned}
$$

$$
\begin{aligned}
K & =T_{3}(E)+T_{1}\left(E_{1}\right)+2 T_{3}\left(E_{1}\right)+2 T_{1}\left(E_{2}\right)+2 T_{3}\left(E_{2}\right)+T_{1}\left(E_{3}\right)+2 T_{3}\left(E_{3}\right) \\
& +T_{1}\left(E_{4}\right)+2 T_{3}\left(E_{4}\right)+2 T_{1}\left(E_{5}\right)+2 T_{3}\left(E_{5}\right)+2 T_{1}\left(E_{6}\right)+2 T_{3}\left(E_{6}\right)
\end{aligned}
$$

\footnotetext{
* It should be noted that Eq. (2l) through Eq. (27) need be calculated only
} once for à particular level scheme of an isotope. 
and $E$ is the incident neutron energy in the c-system (Mev).

$$
\begin{aligned}
& E_{1}=E-0.84 \\
& E_{2}=E-2.1 \\
& E_{3}=E-2.6 \\
& E_{4}=E-2.9 \\
& E_{5}=E-3.1 \\
& E_{6}=E-3.4
\end{aligned}
$$

B. Numerical Results for Known Nuclear Levels $\left(\mathrm{Fe}^{56}\right.$ )

With this ground work, we still need the appropriate transmission coefficients for further numerical results.

$$
\bar{R}=\left(1.25 \mathrm{~A}^{\frac{1}{3}}+0.5\right) 10^{-13} \mathrm{~cm} \text {. }
$$

For $\mathrm{Fe} e^{56}, \overline{\mathrm{R}}=5.2810^{-13} \mathrm{~cm}$.

From Emmerich's Calculations, ${ }^{4}$ we look up the transmission coefficients and plot these versus neutron energy. The calculated values of the transmission coefficients are plotted in Figure 2. It should be noted that these values are calculated for a certain average nuclear potential. Other calculations ${ }^{10}$ are avallable for different physical parameters.

Using Ermerich's calculations as presented in Figure 2, we give the values of $A, B, C, D, \cdot F, H$ and $K$ (as defined in Eq. 21 through Eq. 27) in Table $I$. The calculated inelastic scattering cross sections are given in Table II。: In Table II, $\sigma_{(1)}$ denotes the inelastic cross section for level 1 at a particular energy, $E_{c}$. We have given these energies in Mev and they 


$$
\bar{R}=5.28 \times 10^{-13} \mathrm{~cm}
$$

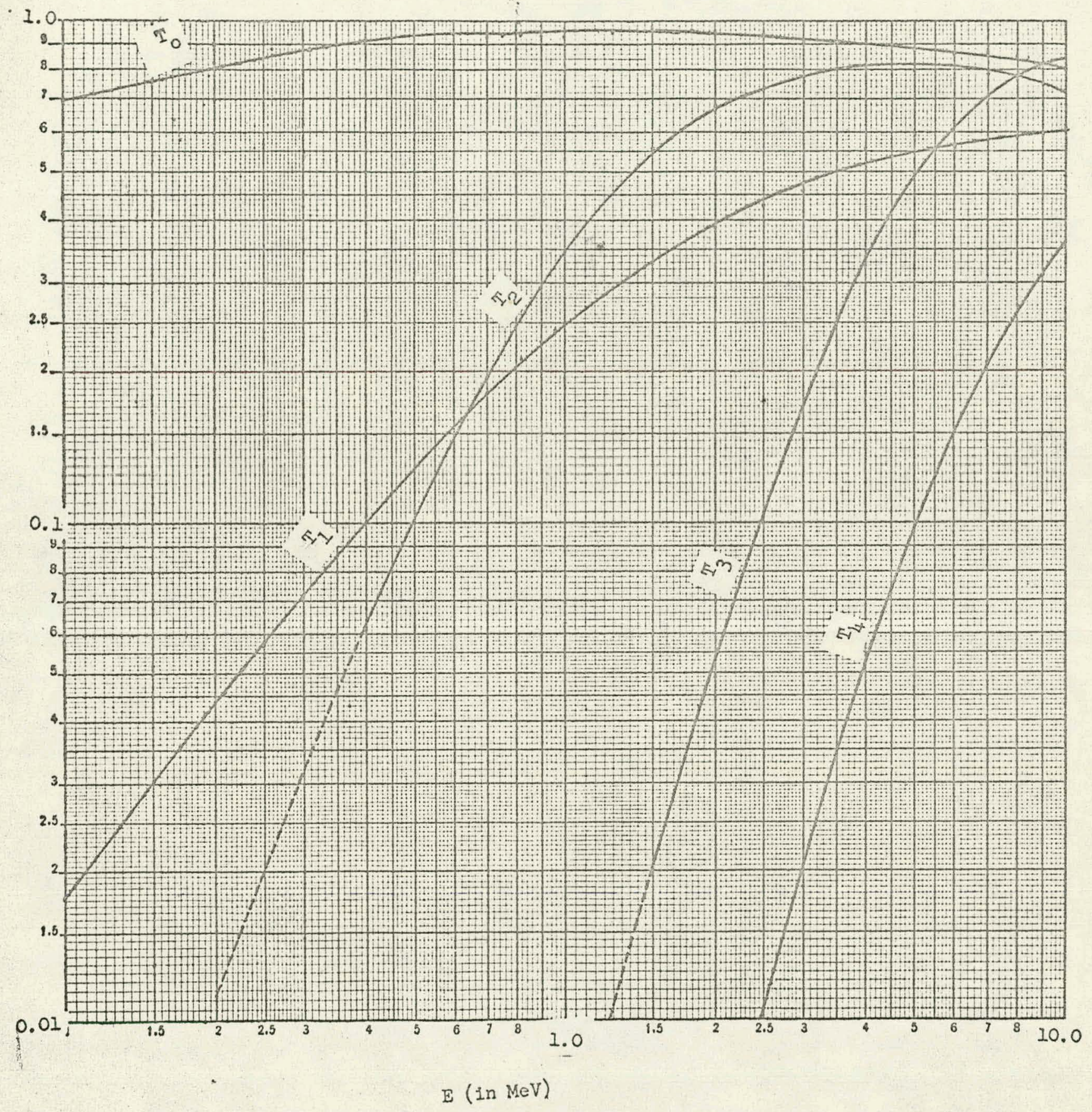

F1g. 2 Transmission Coefficients for $\mathrm{Fe}^{56}$ from Emerich's Calculations. 
are in the center of-mass system. However, for $\mathrm{Fe}^{56}$ the distinction between $\mathrm{E}_{c}$ and $\mathrm{E}_{\mathrm{I}}$ can be ignored.*

\section{Normalization}

At this stage, a comparison of the calculated inelastic scattering cross section (for the various nuclear levels) is made with the existing experimental data. The experimental information ${ }^{18}$ is summarized below.

$\begin{array}{cc}E(\text { Mev }) & F e^{56}\left(n, n^{\prime}\right) \\ \sigma_{\text {in }}(\text { barns }) \\ 1.0 & 0.5 \pm 0.06 \\ 1.2 & 0.6 \pm 0.06 \\ 1.75 & 0.75 \pm 0.05 \\ 2.25 & 1.00 \pm 0.05 \\ 2.45 & 1.10 \pm 0.05 \\ 2.50 & 1.18 \pm 0.05 \\ 2.51 & 1.00 \pm 0.04 \\ 3.25 & 1.21 \pm 0.06 \\ 3.48 & 1.23 \pm 0.07\end{array}$

It may be noted that when the incident neutron energy is $1.75 \mathrm{Mev}$, only the first level (at $0.84 \mathrm{Mev}$ ) can be excited. However, for the incident neutron energy equal to $2.45 \mathrm{Mev}$ both the first and the second levels can be excited. This suggests the normalization procedure for the calculated cross sections in Table II. We normalize $\sigma_{(1)}$ (at $E=1.75 \mathrm{Mev}$ ) to a value of 0.75 barns. $\sigma_{(2)}$ is normalized at $\mathrm{E}=2.45$ to a value given by the difference of 1.10 barns $\left(\sigma_{\text {in }}\left(\right.\right.$ expt) at $E=2.45$ ) and the normalized $\sigma_{(1)}$ at $E=2.45$. The calculated inelastic scattering cross section vs incident energy is given in Figure 3 after this normalization. In Figure 3 , the experimental inelastic scattering cross section versus energy is given; the flags denote the experimental errors.

*This is because $E_{c}=\left(\frac{A}{A+1}\right) E_{L}$ 


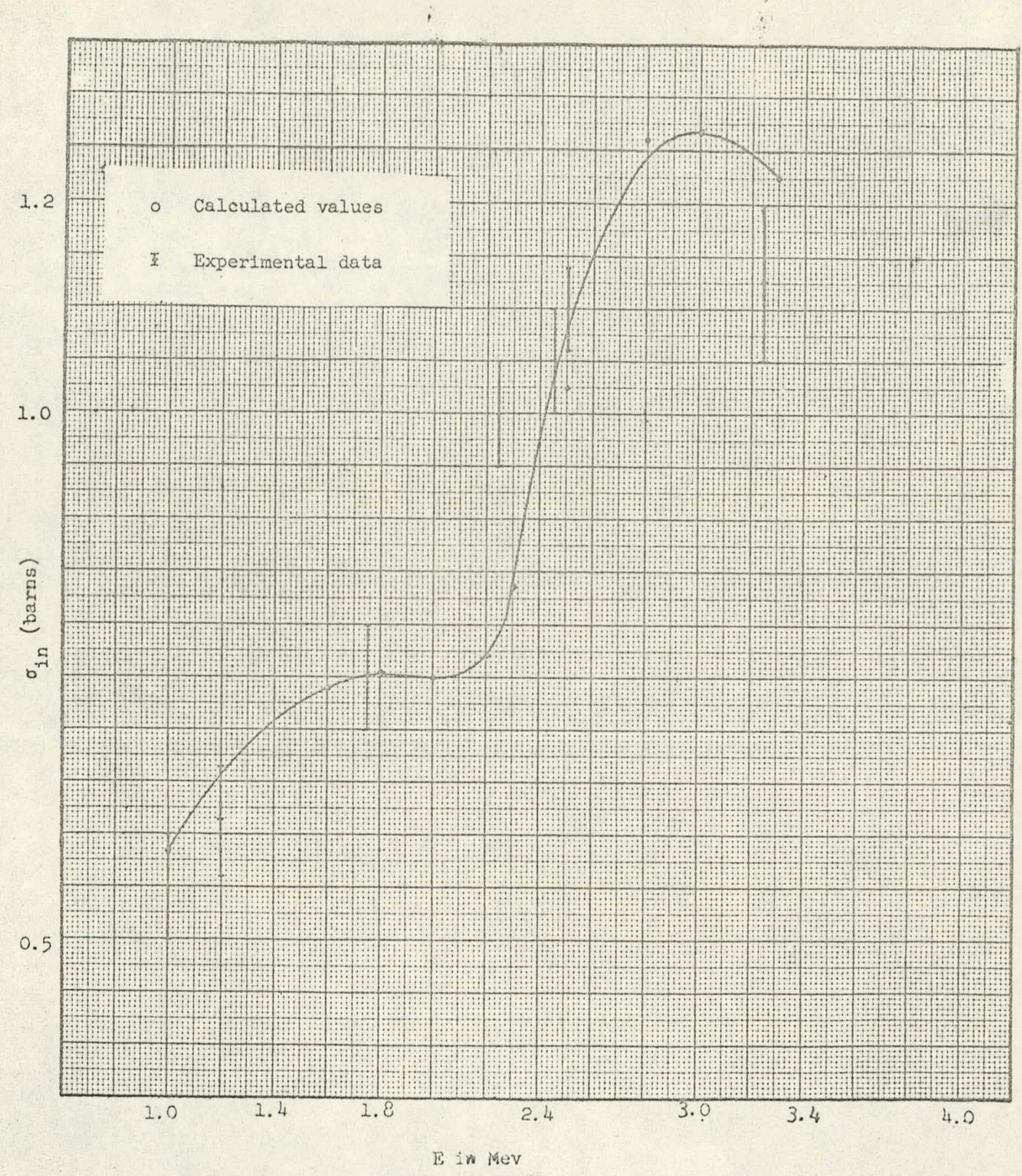

F1g. 3 Comparison of experimental date and calculated cross
section for $\mathrm{Fe}^{56^{2}}\left(\sigma_{1}\right.$ and $\sigma_{2}$ were properly normalized). 
TABLE I THE DENOMHNATOR FACTORS,VS ENERGY (EQS: 21-27) FOR FE: 56

\begin{tabular}{|c|c|c|c|c|c|c|c|c|}
\hline$\dot{E}$ & $x^{2}$ & A & $B$ & $\mathrm{C}$ & $D$ & $\mathrm{~F}$ & H & $\mathrm{K}$ \\
\hline$(\mathrm{Mev})$ & barns & & & & & & & $\because$ \\
\hline .90 & .2303 & .93 & .2475 & .2650 & .980 & .980 & .0350 & .0175 \\
\hline 1.10 & .1884 & .983 & .3310 & .3920 & .1 .3110 & 1.3110 & .1220 & .061 \\
\hline .1 .30 & .1594 & 1.124 & .4150 & .5300 & .1 .5640 & 1.5640 & .230 &. .115 \\
\hline 1.60 & .1296 & .1 .40 & .5400 & .7400 & 1.960 & 1.9600 & .4250 & .225 \\
\hline 1.80 & .1152 & 1.60 & .6100 & .8500 & .2 .220 & 2.220 & .515 & .275 \\
\hline 2.00 & .1036 & 1.76 & .6700 & .9500 & 2.450 & .2 .450 & .609 & .329 \\
\hline 2.20 & .09422 & 1.94 & .7335 & .1 .0570 & 2.660 & 2.660 & .7285 & .436 \\
\hline 2.30 & .09012 & .2 .02 & .7525 & 1.0900 & 2.7710 & 2.7820 & .793 & .517 \\
\hline .2 .50 & .08291 & 2.14 & .8065 & 1.1830 & 2.9610 & 3.0220 & .958 & $\therefore 713$ \\
\hline 2.70 & .07677 & 2.20 & 8655 & 1.2910 & 3.8350 & 3.980 & 1.136 & .9085 \\
\hline 2.80 & .07403 & 2.262 & .9190 & 1.3880 & 4.0870 & $4 . .2820$ & 1.278 & 1.039 \\
\hline 3.00 & .06909 & .2 .442 & 1.0425 & 1.6250 & 5.1420 & 5.4320 & .1 .575 & 1.2925 \\
\hline $3 \cdot 30$ & .06281 & 2.902 & 1.2720 & 2.0640 & 6.0020 & 6.4320 & 2.133 & 1.777 \\
\hline $3: 35$ & .06187 & .2 .98 & 1.3320 & .2 .1850 & 6.120 & 6.5900 & 2.279 & 2.201 \\
\hline 3.42 & .06061 & 3.115 & 1.3840 & 2.2880 & 6.2960 & 6.8120 & 2.423 & 2.373 \\
\hline 3.50 & .05922 & 3.36 & 1.5855 & 2.6810 & 6.6210 & 7.2020 & .2 .861 & 2.77 \\
\hline
\end{tabular}


TABLE II CALCULATED INELASTIC SCATIERING CROSS SECTTON FOR VARIOUS LEVELS OF Fe

\begin{tabular}{|c|c|c|c|c|c|}
\hline $\begin{array}{c}\mathrm{E}_{\mathrm{c}} \\
(\mathrm{Mev})\end{array}$ & $\begin{array}{c}\sigma_{1} \\
\text { barns }\end{array}$ & $\begin{array}{c}\sigma_{2} \\
\text { barns }\end{array}$ & $\begin{array}{c}\sigma_{3} \\
\text { barns }\end{array}$ & $\begin{array}{c}\sigma_{4} \\
\text { barns }\end{array}$ & $\begin{array}{c}\sigma_{5} \\
\text { barns } \\
\end{array}$ \\
\hline .9 & .7725 & & & & \\
\hline 1.1 & .9129 & & & & \\
\hline 1.3 & 1.010 & & & & \\
\hline 1.6 & 1.1536 & & & & \\
\hline 1.8 & 1.1714 & & & & \\
\hline 2.0 & 1.1679 & & & & \\
\hline 2.2 & 1.1428 & .007463 & & & \\
\hline 2.3 & 1.1239 & .02421 & & & \\
\hline 2.5 & 1.0704 & .07052 & & & -. \\
\hline 2.7 & .8878 & .10913 & .1714 & & \\
\hline 2.8 & .8578 & .1339 & .2091 & & \\
\hline 3.0 & .7044 & .1450 & .2257 & .1199 & \\
\hline 3.3 & .5901 & 1713 & .2622 & .1796 & .01348 \\
\hline 3.35 & & & & .1813 & .01617 \\
\hline 3.42 & & & & .1864 & .02272 \\
\hline 3.50 & & & & 1910 & .07891 \\
\hline
\end{tabular}


It should be noted, however, that the calculated inelastic cross sections of the various levels were based on the transmission coefficients as tabulated by Emmerich ${ }^{4}$. If we had used the transmission coefficients tabulated by Beyster et.al. 19 the calculated results would have been different. : This fact-is not surprịing because Emmerich and Beyster et. al have used different nuclear potentials.

D. Calculations of Inelastic Scattering-Probability (Fe $\left.{ }^{56}\right)$ Eincident $\leq 2.86$ Mev)

It is convenient to perform these calculations in the lethargy units. In the following, we will denote by, $u$ and $u$ ' the incident and the exit neutron lethargies respectively. In Figure 4, we give the normalized $\sigma_{(1)}$ : and $\sigma_{(2)}$ versuss lethargy : $u$. Also, $\sigma_{(3)}, \sigma_{(4)}$, and $\sigma_{(5)}$ are plotted. It may be noted that level 6 , cannot make any contributions for incident neutron energy $\leq 2.8$ Mev: We are using the same notation as in the preceeding sections, i.e. $\sigma_{(i)}$ denotes the inelastic scattering cross section for a nuclear level, designated by. 1. Similarly in Figure 5 we plot $\sigma_{(i)}$ : vs lethargy corresponding to exit-neutron energies. Here, some care must be used. The important point to remember is that

$$
E_{\text {exit }}=E_{\text {incident }}-E_{i}
$$

where $\mathrm{E}_{i}$ is the excitation energy, of a nuclear level designated by subscript $i$. Thus for the various levels, Eexit (and therefore $u$ ) is not the same for a fixed incident neutron llethargy, u.

The inelastic scattering probability from group $i$ to group. $j_{j} . P_{j, j}$, is given by Eq. (11)

$$
P_{1, j}:=\frac{\sum_{k} \bar{\sigma}_{k}(i, j) \Delta u_{j, i}}{\sum_{k} \sum_{j} \bar{\sigma}_{k}(i, j), \Delta u_{j, i}}
$$




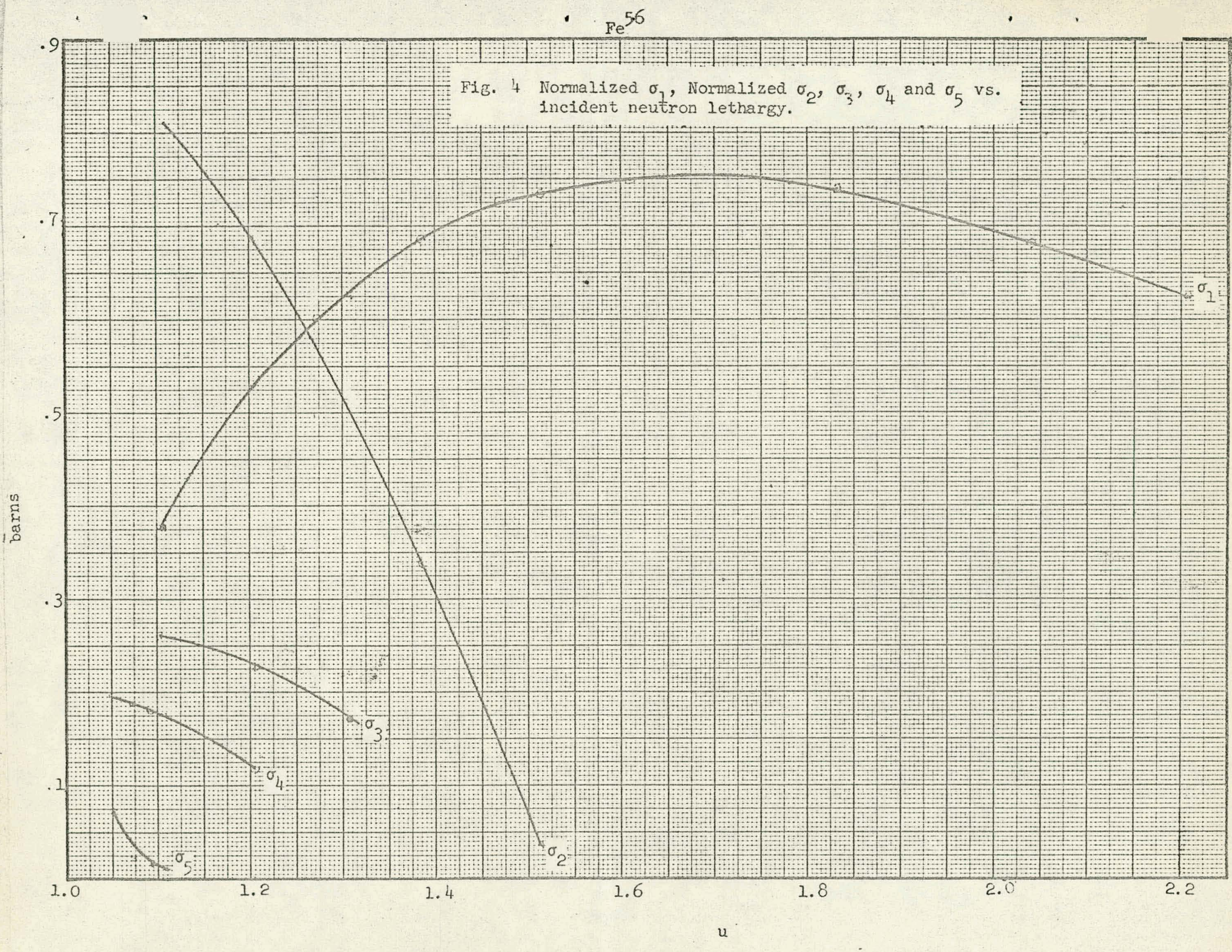




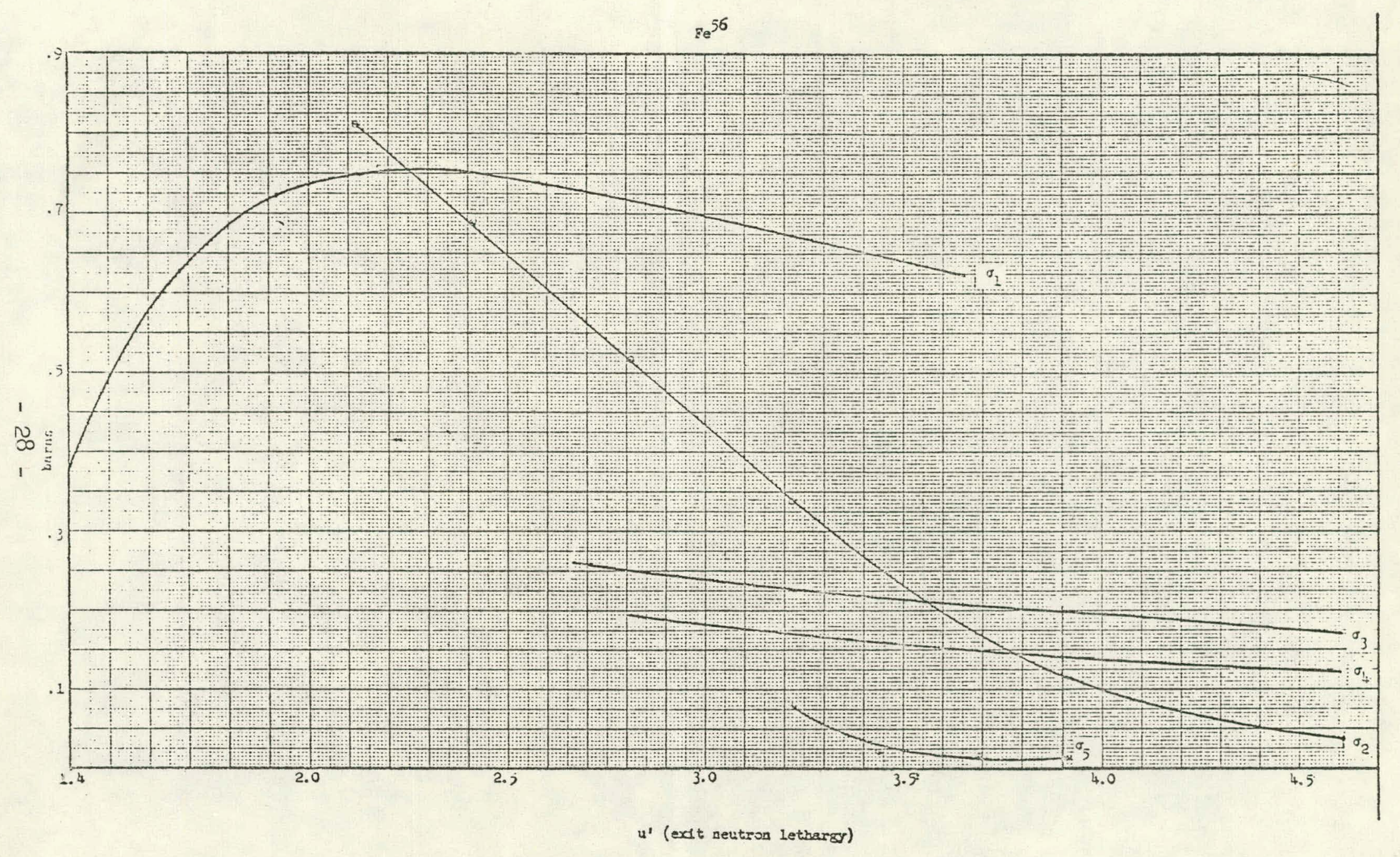
F18. 5 Horrallzed $\sigma_{1}$, normallzed $\sigma_{2}, \sigma_{3}, \sigma_{4}$ and $\sigma_{5}$ r.s. 
where

$$
\begin{aligned}
\mathrm{k}= & \text { summation index for the nuclear levels } \\
\tilde{\sigma}_{\mathrm{k}}(i, j)= & \text { average inelastic scattering cross section (for neturon incident } \\
& \text { in group } i \text { ) from nuclear level designated by the subscript } \mathrm{k} \text { so } \\
& \text { that the exit neutrons are in group } j \text {. The bar denotes an } \\
& \text { average over exit neutron lethargy interval, permissible by } \\
& \text { energy conservation. ( } \left.i \text {.e. } \Delta_{j, j}\right) \text {. } \\
u_{j, i=}= & \text { permissible lethargy interval of exit neutrons in group } j \text { for } \\
& \text { neutrons incident in group } i \text {. }
\end{aligned}
$$

It should be noted that strict normalization condition

$$
\sum_{j} P_{i, j}=1.00000
$$

must be satisfied.

For the sake of clarity and completeness, we present the details of the calculations for neutrons incident in MUF'T energy-group 6 . It may be recalled that energy group 6 has $2.23 \mathrm{Mev}$ and 2.86 Mev as the lower and the upper energy limits. The following steps were followed:

(i) We can see from the level scheme in Fig. 1 that only level 1 , level 2, and level 3 can be excited when the incident neutron energy lies in group 6. It can be immediately seen that for nuclear level 4 to be excited, incident neutron energy must be greater than 2.9 Mev。

(ii) We calculated the permissible ranges of exit neutron energies for these levels. For example, the energy range is $1.39(=2.23-0.84)$ Mev to $2.02(=2.86-0.84)$ Mev for nuclear level 1. Similarly, the permissible exit neutron energy ranges are from $0.12 \mathrm{Mev}$ to $0.76 \mathrm{Mev}$, and $0.76 \mathrm{Mev}$ to $0.0 \mathrm{Mev}$ for nuclear levels 2 and 3 respectively. Since it is most convenient to perform the calculations in lethargy units, these energy limits were converted to 
lethargy limits by using the familiar formula

$$
u=\ln \frac{10}{E}, E \text { is in Mev。 }
$$

(iii) In Fig. 6(a) and Fig。6(b) the inelastic scattering cross sections (normalized, wherever necessary) are plotted as a function of exit neutron lethargy. These curves are relevant only for incident neutrons in group 6. The thick lines represent the permissible ranges of lethargies for the various levels. The dotted (vertical) lines represent the boundaries of the MUFT energy groups. It can be seen from Fig. 6(a) that the inelastic scattering cross section (and hence, the inelastic scattering probabilities) are identically zeros for the energy group $9(u=2.0$ to $u=2.25)$ and the energy group 10 $(u=2.25$ to $u=2.50)$. This result is not surprising because one can arrive at this conclusion by purely energy-conservation arguments. It may be noted that the calculated values of $\sigma_{j}$, where $j$ specifies a nuclear level, are sometimes extrapolated for large values of $u^{2}$ (and up to a maximum value of $u^{2}=7.5$ ).

(iv) It will be convenient to define

$$
D=\sum_{j=7}^{8} \bar{\sigma}_{1}(6, j) \Delta u_{j, 6}^{\prime}+\sum_{j=11}^{18} \bar{\sigma}_{2}(6, j) \Delta u_{j, 6}^{n}+\sum_{j=15}^{23} \bar{\sigma}_{3}(6, j) \Delta u_{j, 6}^{\prime \prime}
$$

We have used primes over the permissible ranges of lethargy because these may be different for each level. It should be noted that $\Delta u_{j, 6}$ depends on the lethargy limits of group $j$.

$$
\text { For groups } j \leq 6, P_{i j}=0
$$

For group j given by $7 \leq j \leq 8$, we have by using Eq. (28) and the above definition of $D$.

$$
P_{6, j}=\frac{\bar{\sigma}_{1}(6, j) \Delta u^{p}{ }_{j, 6}}{D}
$$




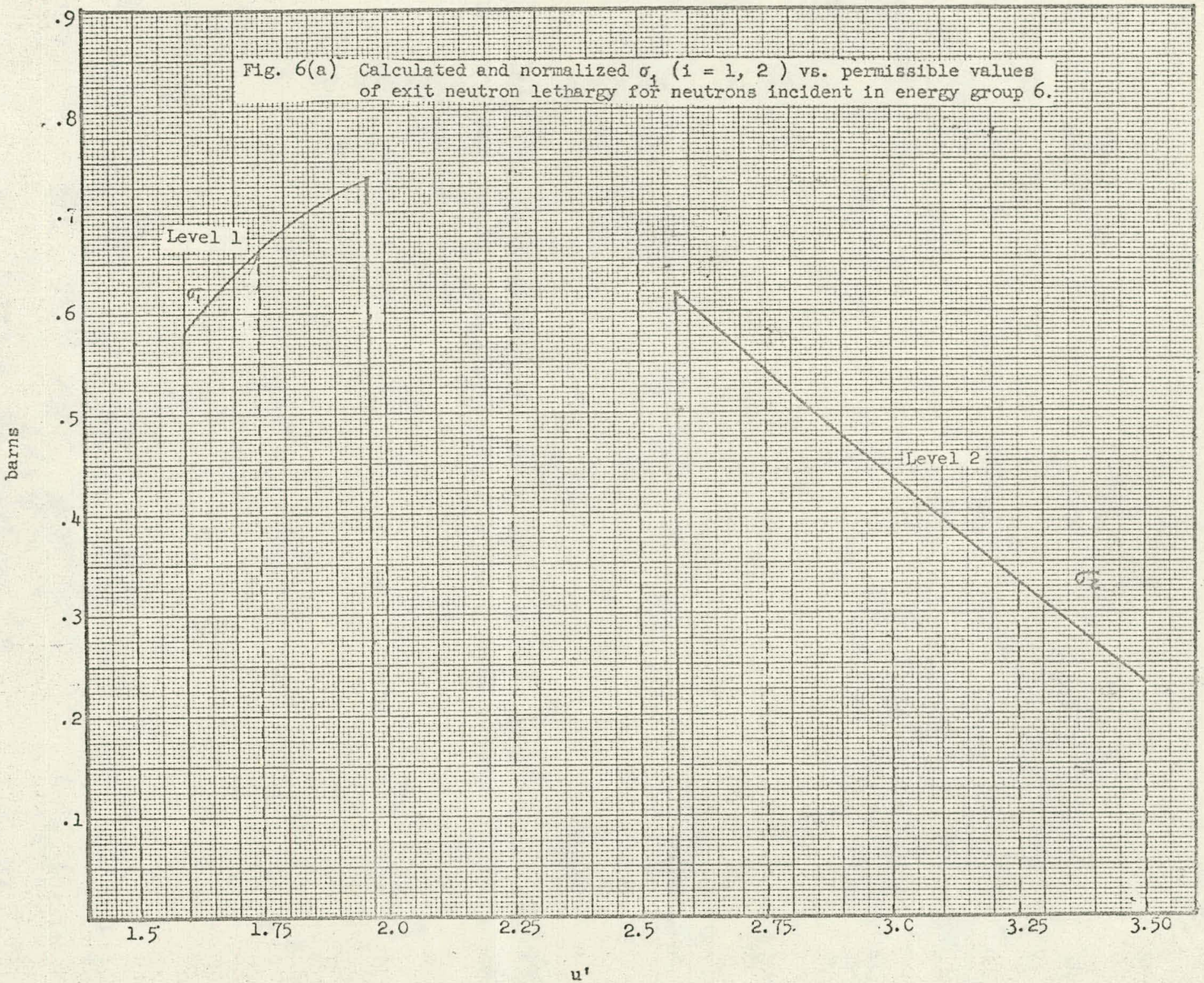




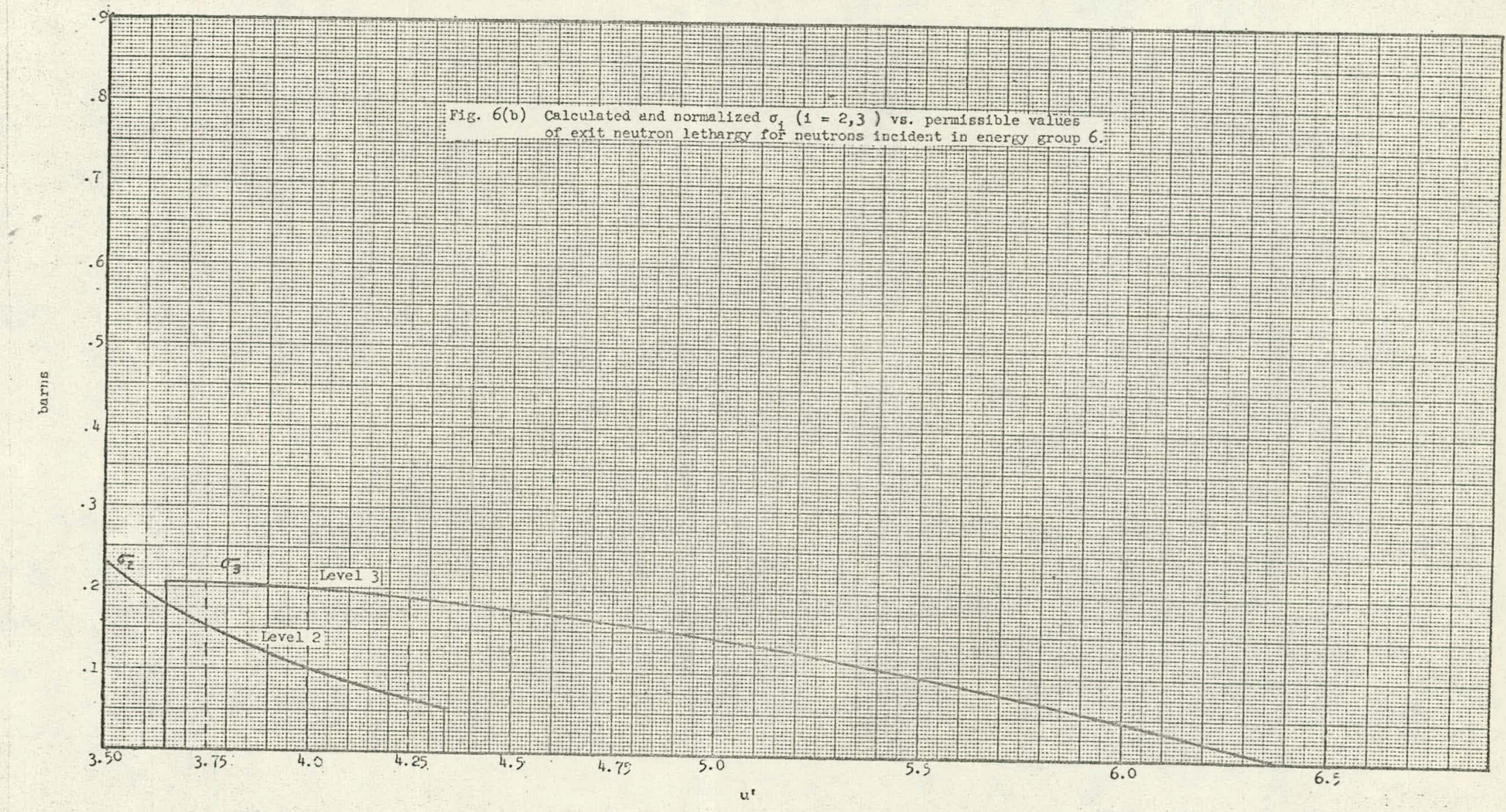


Similarly;

$$
\begin{aligned}
& P_{6, j}=\frac{\bar{\sigma}_{2}(6, j) \Delta a_{j} \sigma}{D} \text { for } 11 \leq j \leq 14 \\
& P_{6, j}=\frac{\bar{\sigma}_{2}(6, j) \Delta \mathrm{i}_{j, 6}+\bar{\sigma}_{3}(6, j) \Delta \mathrm{u}_{j, 6}}{D} \text { for } 15 \leq j \leq 18,
\end{aligned}
$$

and,

$$
P_{6, j}=\frac{\bar{\sigma}_{3}(6, j) \Delta u_{j} 6}{D} \text { for } 19 \leq j \leq 23
$$

The results of our calculations are presented in Table III.

E. Calculation of Tnelastic Scattering Matrix (Fe ${ }^{56}$. E incident $>2.86$ Mev. Above 2.86 Mev incident neutron energy, it.is appropriate to use the statistical model: I The nuclear temperature, T, is equal to $0.95 \mathrm{Mev}$ corresponding to neutron incident energy. of $7 \mathrm{Mev}$. It may be recalled that .

$$
T=a \cdot \frac{1}{2}
$$

The average nuclear temperature for each MUF' energy group is given by

$$
T_{i}=a \cdot t_{i}
$$

where

$$
t_{i}=\frac{2}{\Delta u_{i}} \cdot\left[E_{1}^{\frac{1}{2}}-\frac{1}{\frac{1}{2}} E_{i-1}^{2}\right]
$$

and $E_{i}$ and $E_{i=1}$ are the upper limit and the lower limit of energy group $i$. For $\mathrm{Fe}^{56}$, we get the following values of the nuclear temperature:

$$
\begin{aligned}
& T_{1}=1.066 \\
& T_{2}=0.9402
\end{aligned}
$$


TABLE IIT INELASTIC :SCATTERING PROBABILITY MATRIX FOR F $\mathrm{Fe}^{56}$

$\mathrm{E}<2.86 \cdot \mathrm{MeV}$

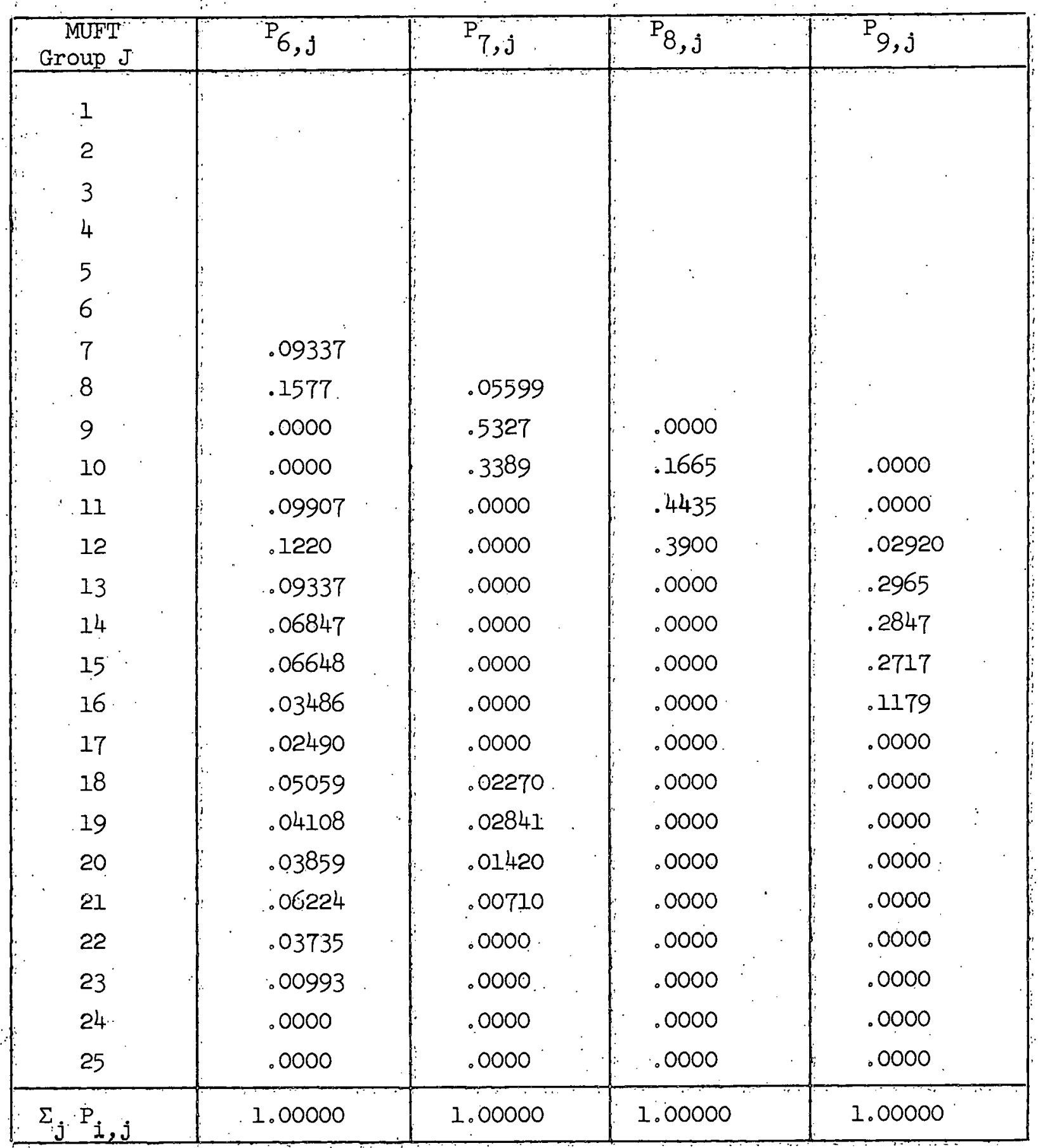

All entries not given above group 9 are zeros. 


$$
\begin{aligned}
T_{3} & \rightleftharpoons 0.8364 \\
T_{4} & =0.7303 \\
T_{5} & \rightleftharpoons 0.6526
\end{aligned}
$$

The inelastic scattering probabilities for $\mathrm{Fe}^{56}$ in the statistical region are given in Table IV. These were calculated from the following:

$$
\cdot P_{i j}=\left(\frac{E_{j-1}}{T_{i}}+1\right) e^{-E_{j-1} / T_{i}}-\left(\frac{E_{j}}{T_{i}}+1\right) e^{-E_{j} / T_{i}}
$$

The entries in Table $V$ are normalized so that

$$
\sum_{j} P_{i j}=1.00000
$$

Thus, for $\mathrm{Fe}^{56}$, the complete inelastic scattering probability matrix is given by. Table III and Table IV: 
TABLE IV INELASTTC SCATHTERING PROBABILITY MATRIX FOR Fe ${ }^{56}$ IN :THE STATISTICAL REGTON ${ }^{*}$

\begin{tabular}{|c|c|c|c|c|c|}
\hline \multirow{2}{*}{$\begin{array}{l}\text { MUFT } \\
\text { Group j }\end{array}$} & $P_{i, j}$ & $P_{2, j}$ & $P_{3, j}$ & $P_{4, j}$ & $P_{5, j}$ \\
\hline & $\mathrm{T}=1.066$ & $\mathrm{~T}=.9402$ & $\mathrm{~T}=.8364$ & $\mathrm{~T}=.7303$ & $T=.6526$ \\
\hline 1 & & & & & \\
\hline 2 & .0712 & & & & \\
\hline 3 & .04225 & .02819 & & & \\
\hline 4 & .07694 & .05926 & .0440 & & \\
\hline 5 & .1104 & .09515 & .07946 & .0606 & \\
\hline 6 & .13122 & .12363 & .1133 & .09749 & .08344 \\
\hline 7 & .13462 & .13594 & .1339 & .1269 & .1187 \\
\hline 8 & .1231 & .13134 & .1369 & .1398 & .1403 \\
\hline 9 & .1028 & .1144 & .1248 & .1351 & .1433 \\
\hline 10 & .08006 & .09209 & .1040 & .1178 & .1305 \\
\hline 11 & .05900 & .06963 & .0809 & .0950 & .1087 \\
\hline 12 & .04165 & .05016 & .05950 & .07185 & .08443 \\
\hline 13 & .02844 & .03479 & .04196 & .05178 & .06211 \\
\hline 14 & .01891 & .02343 & .02862 & .03593 & .04379 \\
\hline 15 & .01233 & .01541 & .01903 & .02420 & .02986 \\
\hline 16 & .00790 & .00996 & .01239 & .05193 & .01985 \\
\hline 17 & .00501 & .00634 & .00795 & .01029 & .01292 \\
\hline 18 & .00315 & .00400 & .00503 & .00656 & .00828 \\
\hline 19 & .00196 & .00250 & .00315 & .00413 & .00525 \\
\hline 20 & .00122 & .00155 & .00197 & .00258 & .00329 \\
\hline 21 & .00122 & .00155 & .00197 & .00259 & .00331 \\
\hline 22 & .00045 & .00059 & .00074 & .00098 & .00126 \\
\hline 23 & .00017 & .00021 & .00028 & .00033 & .00047 \\
\hline 24 & .00006 & .00008 & .00010 & .00011 & .00017 \\
\hline 25 & .00002 & .00002 & .00005 & .00005 & .00007 \\
\hline
\end{tabular}




\section{REFERREITCES}

1. H. Bohl, E. M。 Gelbard, G。H。:Ryan, "MUFT-4 - Fast Neutron Spectrum Code for the IBM-704",. WAPD-TM-7.2 (July 1957).

2. W. Hauser and H. Feshbach, Phys. Rev. 87, 366 (1952).

3. W. S. Fmerich, in Fast Neutron Physics, :Part II, Interscience Publishers (1963).

4. W. S. Emmerich, The Optical Model for Fast Neutron Interactions, W Research Labs 62-118-540-PI (formerly 6-94511-6-R2 \& R-19) 1962.

5. Nuclear Reactions Theory I \& II, Handbuch der Physik, edited by S. Flugge (J. Springer Verlag, Berlin 1959).

6. D. Bodansky, Annual Review of Nuclear Science p. 79 (E. Segre, Ed. Annual Reviews, Inc. Palo Alto, California 1962).

7. H. Feshbach, in Nuclear Spectroscopy, pages 625, 1033. (F. Aizenberg Selove, Ed: Academic Press, New York 1960).

8. M. K. Bannerjee, reference 7 , page 695 .

9. C. A. Levinson, reference 7 , page 670 .

10. T. Ericson, Advances in Physics, 2, 425 (1960).

11. T. Ericson, Proceedings of the International Conference on Nuclear Structure, (D.A. Bromley \& $\dot{E}_{0} \cdot W_{0}$. Vogt, Editors; University of Toronto Press 1960).

12. T. D. Newturn, Canadian J. Physics 34, 804 (1956).

13. A.G.W. Cameron, Canadian J. Phys. 35, 1021 (1957); Canadian J. Physics 36, 1040 (1958).

14. R. W. Jackiw, M。A. Rothman and D. M. Van Patter, Bartal Research Foundation Summary Report, 1961.

15. Do.B. Thomsori, Doctoral Thesis, Oniversity of Kansas, 1960.

16. R. Sherr and F.'P. Brady, Phys.Rev. 124, 1928 (1961).

1\%. D. M. Van Patter and R. W. Jackiw, Proceedings of the International Conference on Nuclear slructure, (1960). 
18. J. R: Beyster, R。 Go Schrandt, Mo Walt and E. Salmi, Los Alamos Scientific Report TA-2099 (1960).

19. H. A. Harvey, Invited Paper at Symposium of Neutron Time of Flights Methods, Saclay (France) 1961 .

20. H. A. Bethe, : Phys. Rev. 20, 332 (1963); Revs: Modern Physics 2, 69 (1937).

21. I. G. Weinberg and Jo M. Blatt, Am. J. Phys. 21, 124 (1953).

22. J. M. Lang and K。 Jo Le Couteour, Proc. Phys. Soc. London A67, 585 (1954); Nuclear Physics 14; 21 (1959).

23. T. Ericson; Proc. International Conference on Nuclear Structure, page 697 (1960).

24. T: D: Newton, Canadian J. Phys. 34 804 (1956); Errata 35, 1040 (1958)。

25. P. F. A. Klinkenberg; Revs. Modern Phys. 24, 63 (1953).

26. A. E. S. Green and D. Fo Edwards, Phys。'Rev。 91,46 (1953).

27. A. G.'W. Cameron, Canadian J. Phys.o 35, 1021 (1957); ibid 36, $1040(1958)$. 
Inelastic Scattering Probabilities in the Statistical Region

In the statistical region (when the incident neutron energy is $>3 \mathrm{Mev}$ ), general results can be obtained for a multigroup fast neutron spectrum code. The numerical results depend upon the nuclear temperature. It is necessary to define an average nuclear temperature for incident neutrons in an energy group in the MUFT IV scheme. $E_{1}$ and $E_{i-1}$ will denote the upper and the lower energy limits of a MUFT group designated by 1 . The average nuclear temperature, $T_{1}$, for energy group is is as follows:

$$
T_{i}=t_{i} a
$$

where

$$
t_{i}=\frac{2}{\Delta u_{i}}\left(E_{i}^{\frac{1}{2}}-E_{i-1}^{\frac{1}{2}}\right)
$$

In Eq. $(A-1), \Delta u_{i}$ is the lethargy interval for energy group $i$. The probability of neutron inelastic scattering incident in an energy group 1 to an energy group $j$ is denoted by $P_{i, j^{\circ}}$

$$
P_{i, j}=\left(E_{j-1} / T_{i}+1\right) e^{-E_{j-1} / T_{i}}-\left(E_{j} / T_{i}+1\right) e^{-E_{j} / T_{i}}
$$

It should be noted that all references in Eq. (A-2) to the incident energy group 1 come through $T_{i}$. This, genermal results can be computed as a function of the values of the nuclear temperatures.

For the purposes of convenience, $t_{i}$ in $E q .(1-1)$ is tabulated in Table A-I for MUFT energy groups. Once the value of the constant a is known, the approprlate average nuclearr temperatures can be immediately calculated.

In Figures $A-1$ through $A-5$, the values of inelastic scattering probabilities are given as a function of the nuclear temperature. The calculated values are also given in Tables A-II and A-III. 


\begin{tabular}{|c|c|c|c|}
\hline $\begin{array}{l}\text { Energy } \\
\text { Group }\end{array}$ & $\begin{array}{l}\text { Lower. Limit of } \\
\text { Energy Group }\end{array}$ & $\begin{array}{l}\text { Upper Limit of } \\
\text { Lethargy }\end{array}$ & $t_{i}=\frac{2}{\Delta U_{i}}\left(E_{i}^{\frac{1}{2}}-E_{i-1}^{\frac{1}{2}}\right)$ \\
\hline 1 & $7.79 \mathrm{Mev}$ & 0.25 & 2.9698 \\
\hline 2 & 6.07 & 0.50 & 2.6186 \\
\hline$\ldots 3$ & 4.72 & 0.75 & 2.3294 \\
\hline 4 & 3.68 & 1.00 & 2.0338 \\
\hline 5 & 2.86 & 1.25 & 1.8174 \\
\hline 6 & 2.23 & 1.50 & 1.5827 \\
\hline 7 & 1.74 & 1.75 & 1.3938 \\
\hline $8^{\circ}$ & .1 .35 & 2.00 & 1.2576 \\
\hline-9 & 1.05 & 2.25 & 1.0976 \\
\hline 10 & .821 & 2.50 & . 9488 \\
\hline 11 & .639 & 2.75 & .8537 \\
\hline 12 & .498 & 3.00 & . 7495 \\
\hline 13 & .388 & 3.25 & .6624 \\
\hline 14 & .302 & 3.50 & .5868 \\
\hline 15 & .235 & 3.75 & .5182 \\
\hline .16 & .183 & 4.00 & .4559 \\
\hline 17 & .143 & 4.25 & .3970 \\
\hline 18 &. .111 & 4.50 & . 3599 \\
\hline 19 & .0865 & 4.75. & .3125 \\
\hline 20 & $.06 \% 4$ & 5.00 & .2759 \\
\hline 21 & .0409 & 5.50 & . 2295 \\
\hline 22 & .0248 & 6.00 & .1790 \\
\hline$? 3$ & $\therefore 015$ & 6.50 & $\therefore 1400$ \\
\hline 24 & .00912 & 7.00 & .1079 \\
\hline $25^{*}$ & .00553 & 7.50 & - \\
\hline
\end{tabular}

* Since energy group 25 is the last group for which inelastic scattering probability is entered and no inelastic scattering within a group is allowed, we do not need $t_{25^{\circ}}$ 


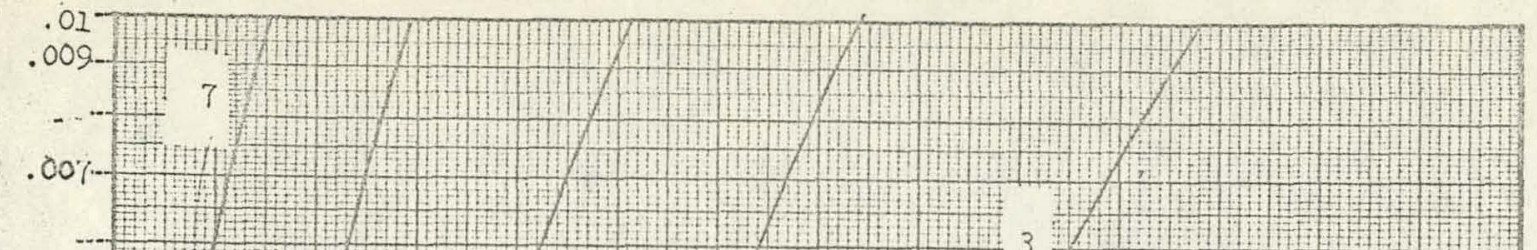

.005 (1)

-

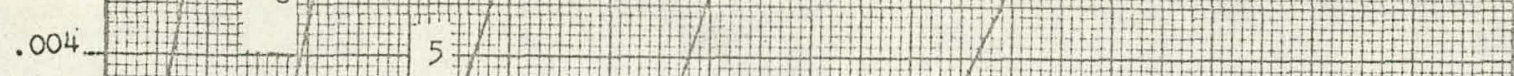
(E+)

.003 H. H. (4) \begin{tabular}{|l|l|l|l|l|l|}
$.002 .-1$ & \\
\hline
\end{tabular} (H)

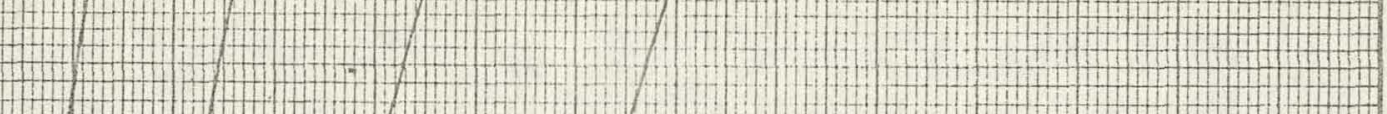

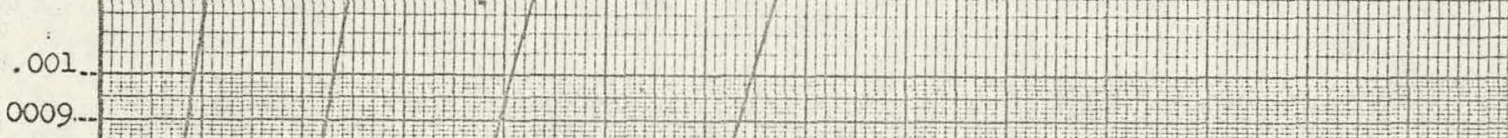
.009

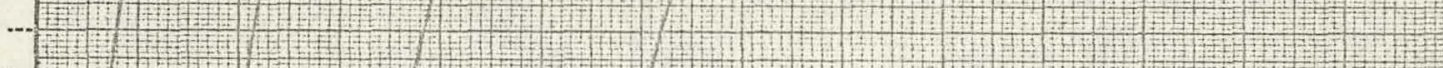

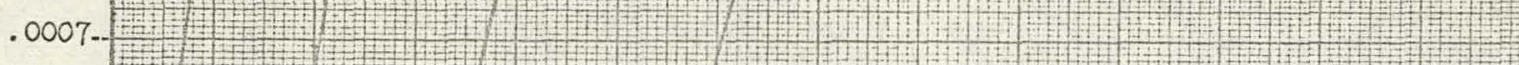
In:

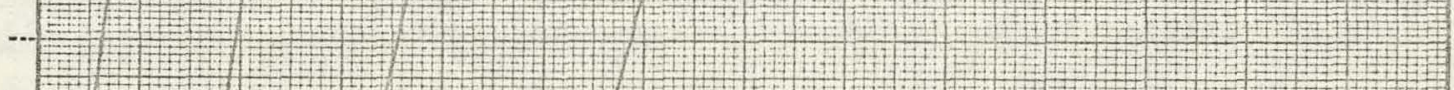
.0005

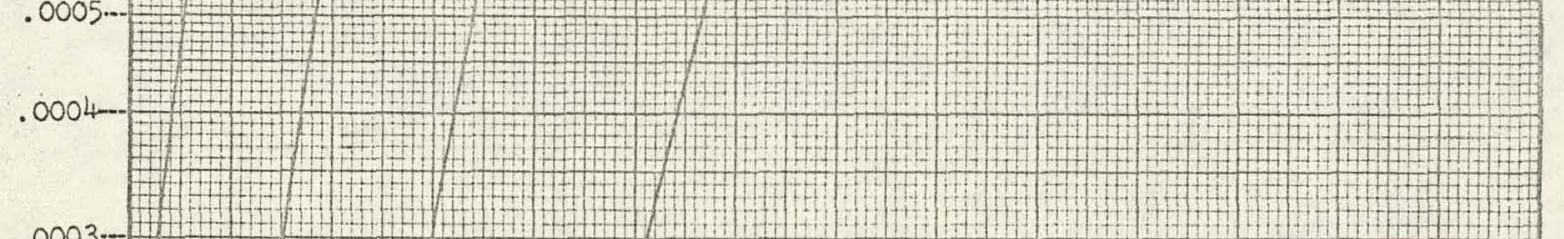
$.0003-2$

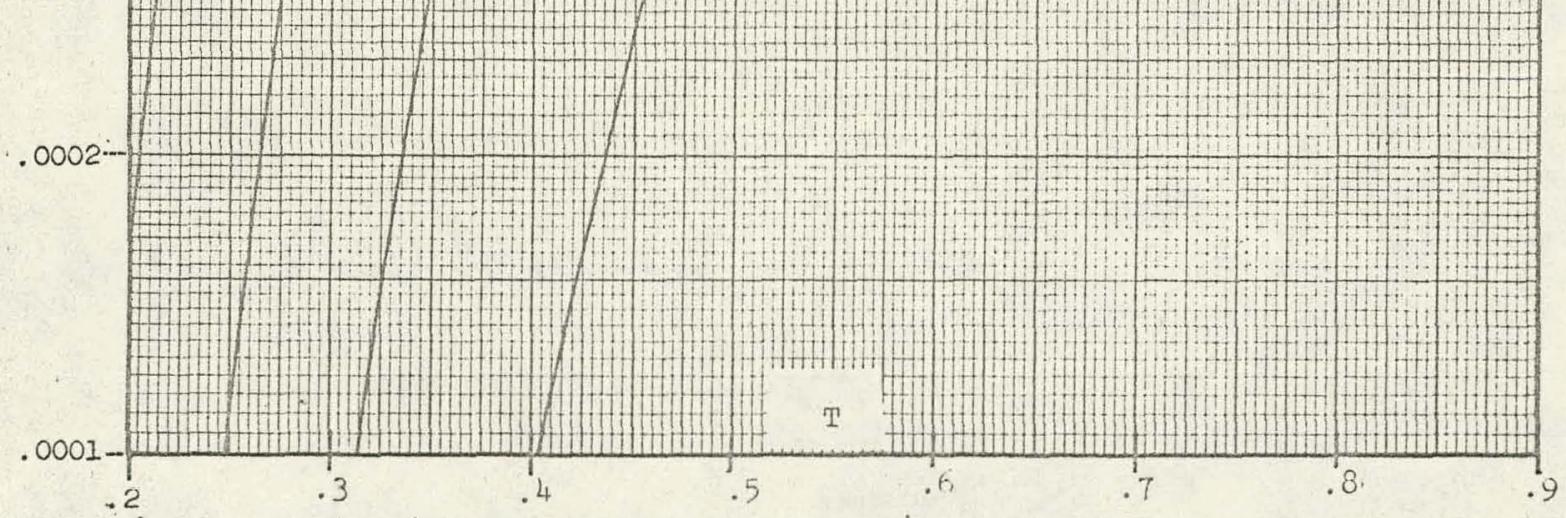

Fig.A-I Calcilated Inelastic Scattering Probabilities for Various MUFT Groups versus Nuclear Temperature 


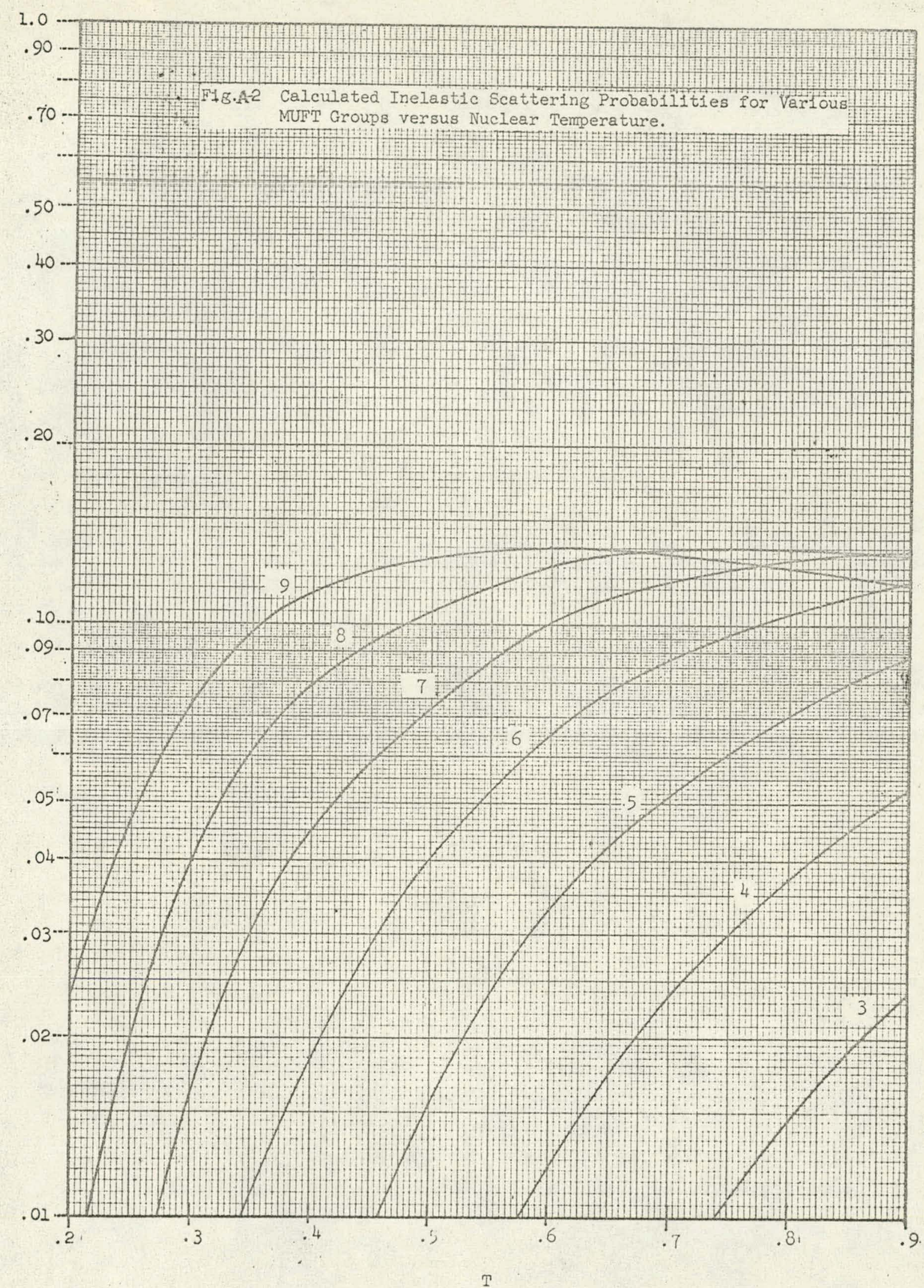


1.0

.90

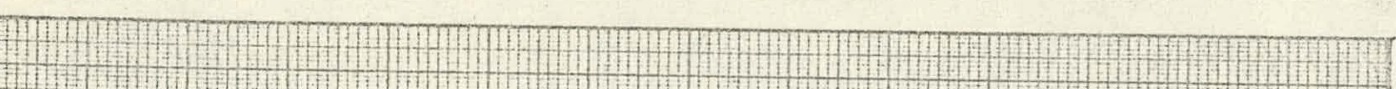

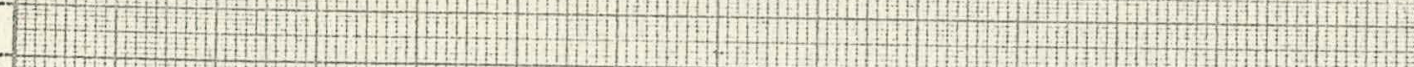

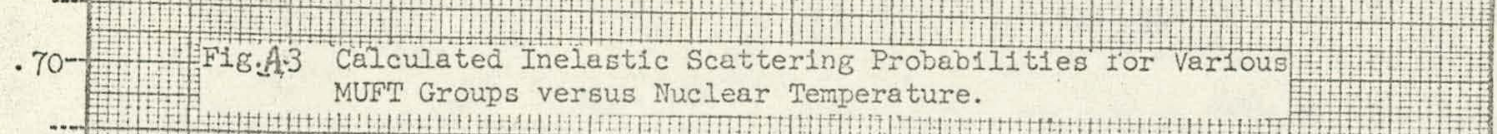

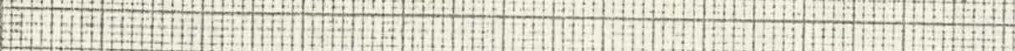

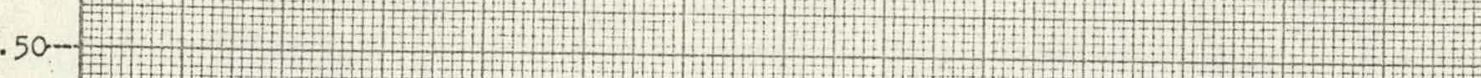

40.-2 +

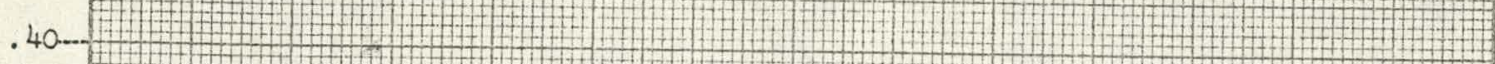

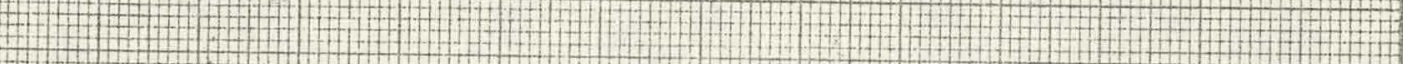

H.

.30-

H.

-

.20 .

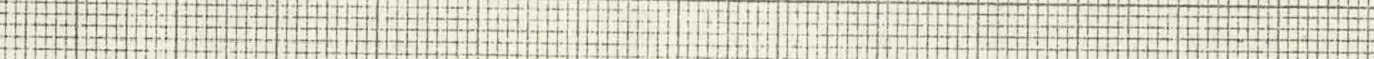

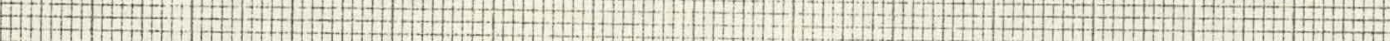

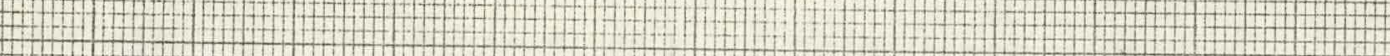

$\cdot$

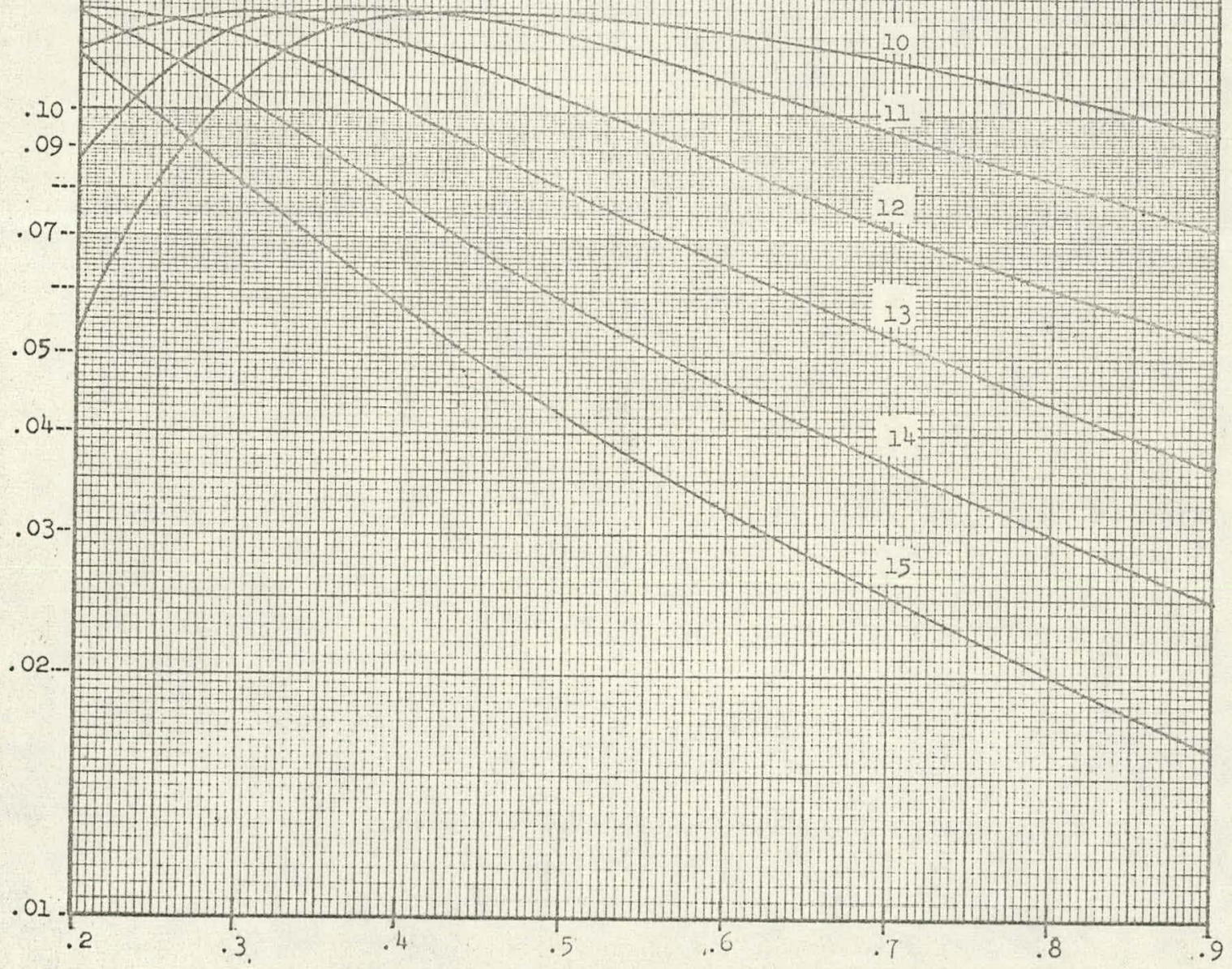




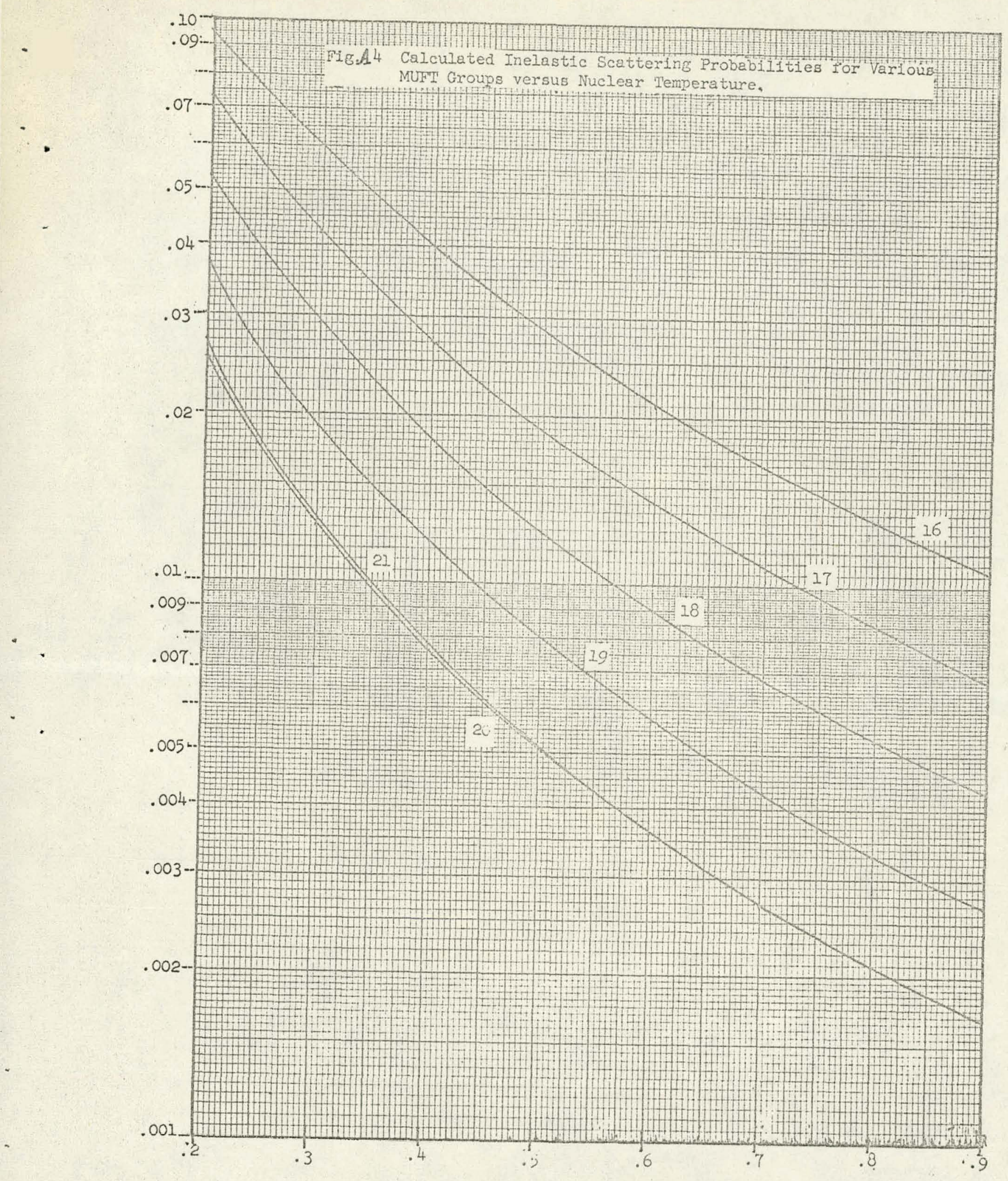


.009

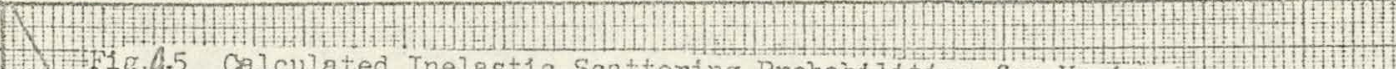
N W 1 -5 Calculated Inelastic Scattering Prohabilities for Various

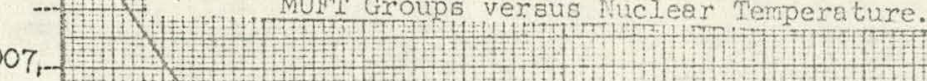

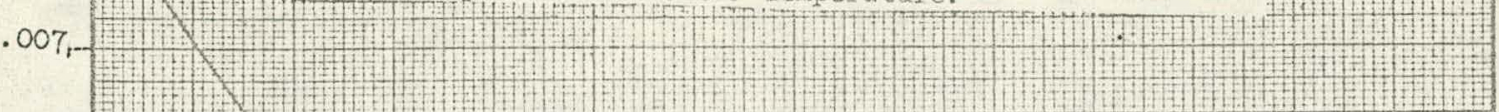
(B)

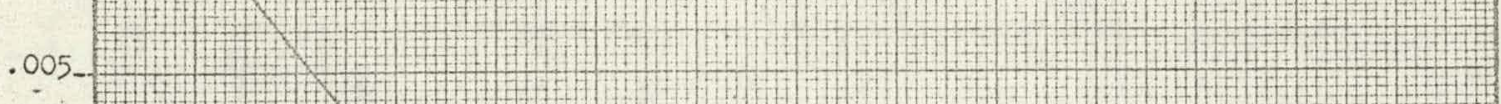

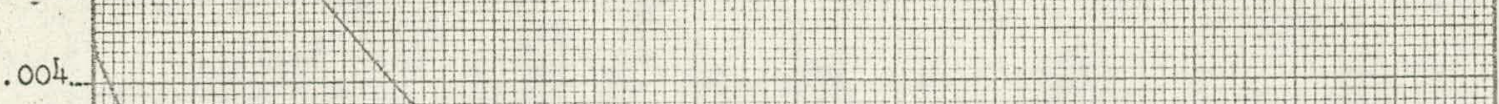
X.

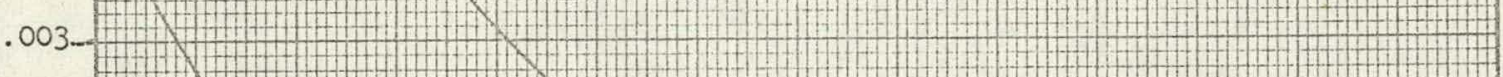

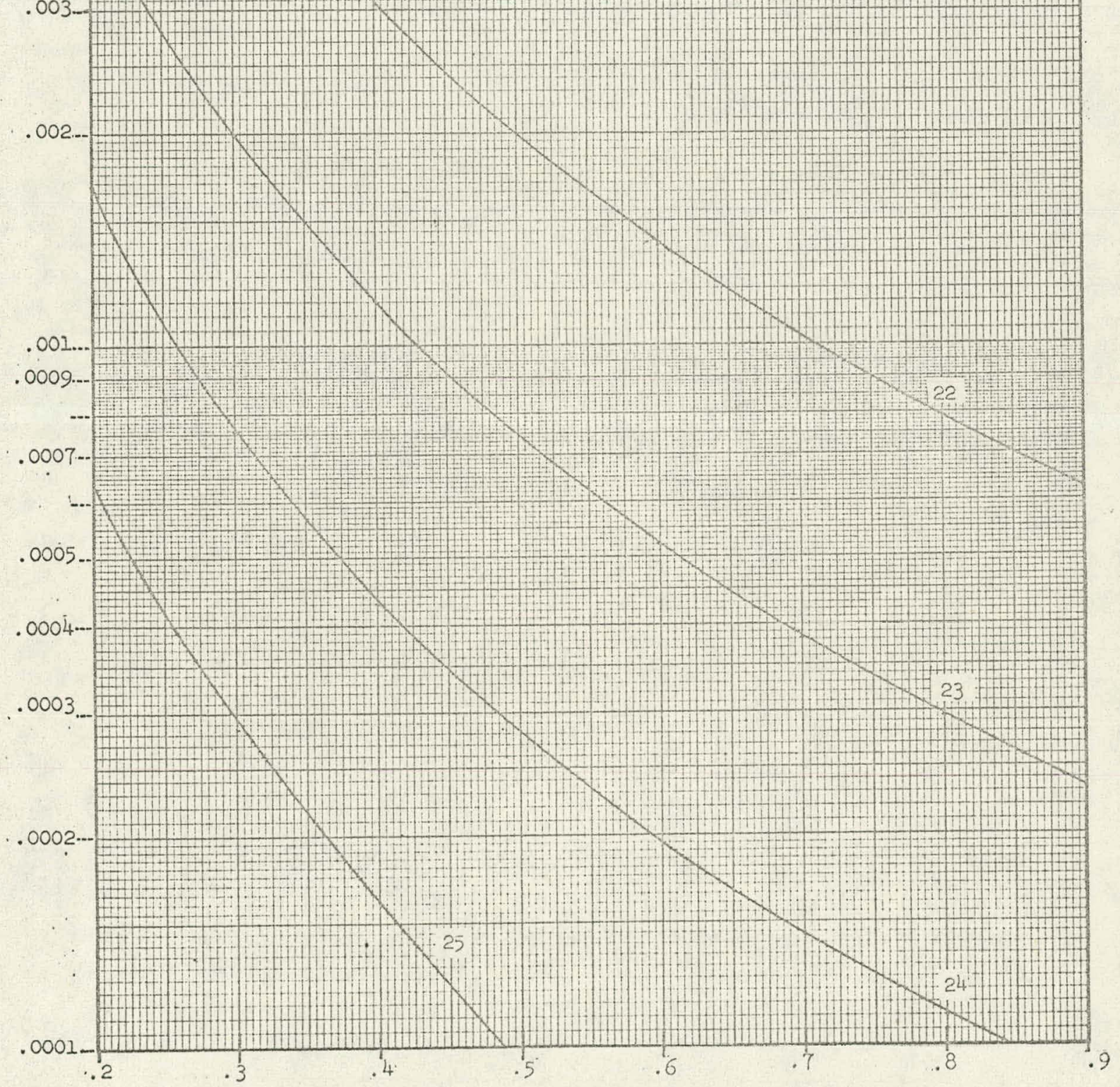


TABLE A-IT: NORMALIZED INELASTIC SCATTERTNG PROBABILITIES

\begin{tabular}{|c|c|c|c|c|c|c|}
\hline - MUFT GROÜP & $T=.2$ & $T=: 25$ & $T=.27$ & $T=.30$ & $T=: 35$ & $T=.4$ \\
\hline 1 &. .00 & .00 & .00 & .00 & .00 & .00 \\
\hline 2 & .00 & .00 & .00 & .00 & .00 & .00 \\
\hline 3 & .00 & .00 & .00 & .00 & .00 & .00 \\
\hline 4 & $: 00$ & .00 & .00 & .00 & .0003 & .0009 \\
\hline 5 & .00 & .0001 & $: 0003$ & .0007 & .0022 & .0053 \\
\hline 6 & :0002 & .0012 & .0021 & .0042 & .0100 & .0185 \\
\hline .7 & .00 .1 .5 & .0063 & .0095 & .0158 & .0291 & .0445 \\
\hline 8 & .0073 & .0210 & .0281 & .0398 & .0602 & $: 0794$ \\
\hline 9. & .0233 & .0484 & .0589 & .0739 & .0956 & .1120 \\
\hline 10 & .0520 & $: 0836$ & .0943 & .1077 & .1232 & .1314 \\
\hline 11 & .0874 & .1151 & .1223 & .1294 & .1345 & .1334 \\
\hline 12 & .1178 & $\therefore 1326$ & $.13 \frac{13}{3}$ & .1341 & . 1292 & $.12 i 1$ \\
\hline 13 & .1335 & .1327 & .1296 & .1234 & $\therefore 1122$ & .1005 \\
\hline 14 & .1318 & .1190 & .1128 & .1038 & .0899 & .0779 \\
\hline .15 & $\therefore 1168$ & .0978 & .0908 & .0812 & .0678 & .0572 \\
\hline 16 & .0953 & .0752 & .0686 & .0601 & .0488 & .0402 \\
\hline 17 & .0729 & .05 .49 & .0494 & .0425 & .0338 & .0274 \\
\hline 18 & .0530 & .0385 & .0343 & .0291 & .0227 & .0182 \\
\hline 19 & .0370 & .0261 & .0231 & .0194 & .0149 & .0118 \\
\hline 20 & .0250 & $: 0173$ & .0152 & .0126 & .0096 & $: 0076$ \\
\hline 21 & .0272 & .0184 & $\therefore 0161$ & .0138 & .0100 & .0078 \\
\hline$" 22$ & $: 0112$ & .0074 & .0064 & .0052 & .0039 & .0030 \\
\hline 23. & .0044 & .0029 & .0025 & .0020 & .0013 & .0012 \\
\hline 24 & .0017 & .0011 & .0009 & .0008 & .0006 & .0005 \\
\hline .25 & .0007 & .0004 & .0005 & .0005 & .0002 & .0002 \\
\hline
\end{tabular}


TABLE A-III: NORMALIZED INELASTIC SCATWERING PROBABILITIES

\begin{tabular}{|c|c|c|c|c|c|}
\hline MUFT Group & $T=.5$ & $T=: 6$ & $T=.7$ & $T=.8$ & $T=9$ \\
\hline 1 & .00 & .00 & .00 & .00 & .0015 \\
\hline 2 & .00 & $\therefore 00$ & .00 & .00 & .0075 \\
\hline 3 & .0008 & .0029 & .0074 & .0145 & $: 0237$ \\
\hline 4 & .0045 & .0121 & $\therefore 0236$ & .0377 & $: .0525$ \\
\hline 5 & .0165 & .0332 & $.052 \%$ & .0715 & .0880 \\
\hline 6 & .0411 & .0658 & .0880 & .1059 & .1182 \\
\hline 7 & .0755 & .1007 & .1183 & .1291 & $: 1336$ \\
\hline 8 & 1089 & 1261 & :I337 & : 1350 & $: 1316$ \\
\hline 9 & .1302 & .1348 & .1319 & .1254 & : 1166 \\
\hline 10 & .1341 & .1272 & $: 1169$ & : 2060 & ח959 \\
\hline 11 & $\therefore 1229$ & 1088 & .0953 & .0833 & .0725 \\
\hline 12 & .1029 & .0864 & .0727 & .0619 & .0527 \\
\hline .13 & .0802 & .0646 & .0529 & .0439 & .0367 \\
\hline 14 & .0592 & .0462 & .0369 & .0301 & .0248 \\
\hline 15 & .0419 & .0318 & .0249 & .0201 & .0164 \\
\hline 16 & .0286 & .0213 & .0164 & .0132 & .0106 \\
\hline 17 & .0190 & .0140 & .0107 & .0084 & .0068 \\
\hline 18 & .0124 & .0090 & .0068 & .0053 & $: 0043$ \\
\hline 19 & .0080 & .0057 & .0043 & .0033 & .0027 \\
\hline 20 & .0050 & .0036 & .0027 & .0021 & .0017 \\
\hline 21 & .0051 & .0036 & .0027 & .0021 & .0017 \\
\hline 22 & .0020 & .0014 & .0010 & .0008 & .0006 \\
\hline 23 & .0007 & .0005 & .0004 & 0003 & .0002 \\
\hline 24 & .0003 & .0002 & .0001 & .0001 & .0001 \\
\hline $\begin{array}{r}25 \\
\ldots\end{array}$ & .0002 & .0001 & .00 & .00 & .00 \\
\hline
\end{tabular}




\section{APPEIVDIX $\cdot B$ \\ Summary of Nuclear Level Density}

It would be helpful for the purpose of a discussion to list some experimental points concerning the nuclear level density:

(1) For a given nucleus, the nuclear level spacing decreases rapidly with the increase in the excltation energy.

(ii) For a fixed excitation energy the level spacing decreases rapidly with an increase in the mass number $A$.

-(iii) It has been found that the nuclear level density depends, at least, on the following three effects:

(a) Magic Nuclei: :It has been observed that the level spacing increases by several orders of magnitude, when the number of neutrons and, or, protons are magic. In nuclear physics, a magic number is denoted by the following: $8,28,50,82$ and. 128. This effect can be seen in Fig. B-1. In this figure, the level spacing per spin state is plotted versus the number of neutrons in compound nucleus.

(b) Odd-Even Effect: The nuclear level spacing (or the nuclear level density) is also influenced by, the number of neutrons being odd and the number of protons being even, or other combinations. For example, one would expect that the level spacing of an even-even nucleus is different from those of an odd-even nucleus.

(c) Pairing Effect: In a highly successfur model of a nucleus the nuclear shell model - the neutrons (and the protons) prefer to occupy states in pairs consistent with the Pauli's exclusion principle. It has been proposed that an additional energy. is required to break a pair, so that one of the nucleons can be excited to a higher level. In other words, the excitation energy effectively becomes $E_{n}-\delta$ rather than $E_{n}$, where $\delta$ is the pairing energy. 


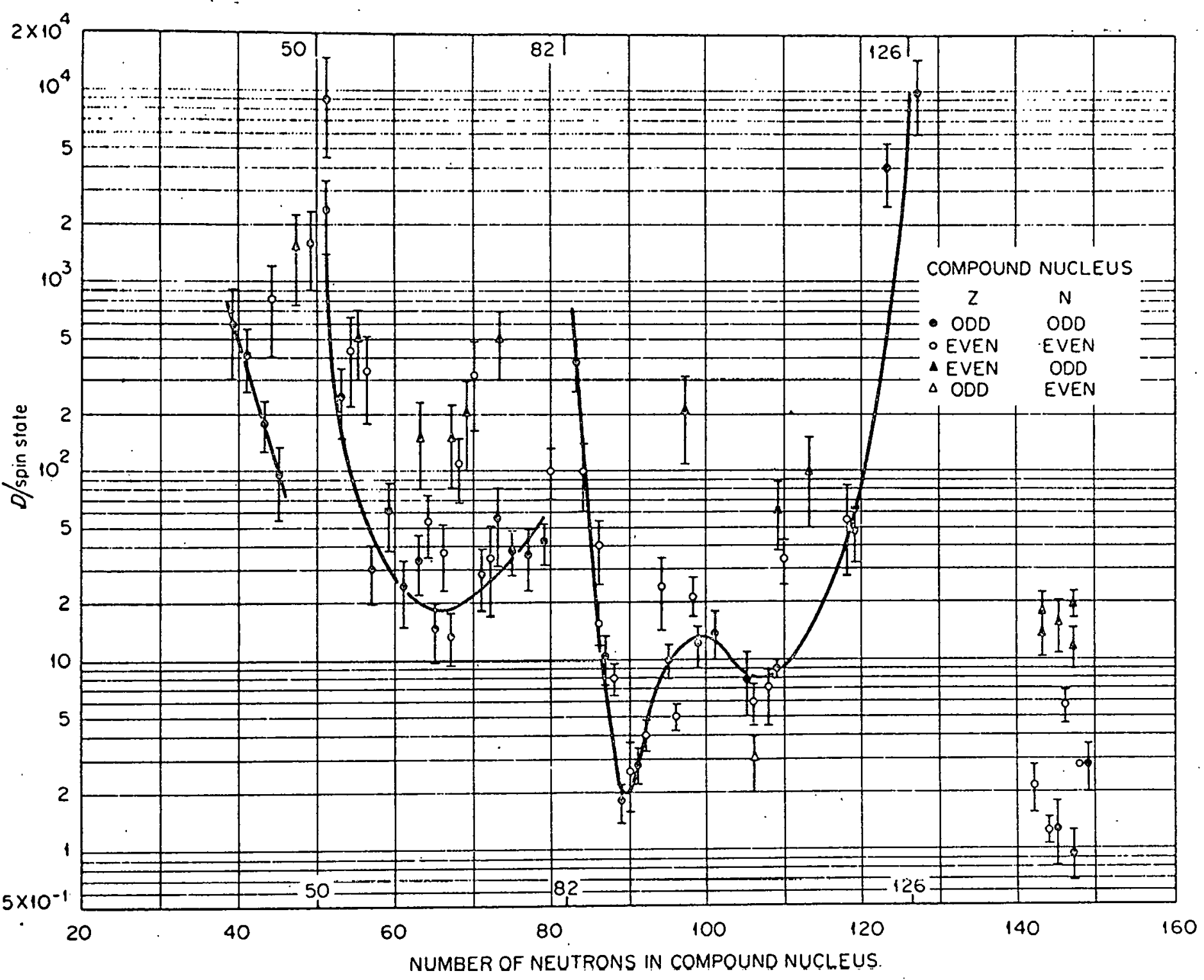

Fig. B-I. Level Spacing per Spin State Versus Number of Neutrons in Compound Nucleus. (Harvey 1961). 
The calculation of the inelastic scattering probability depends upon the number of nuclear levels (and on the assignment of their spins and parities), which lie below an excitation energy - the energy of the incident neutron. All this information being not always available; there arises a need for a theoretical description of nuclear levels by a nuclear model. A comparison of theoretical calculations and experimental data is generally made for a justification of a particular nuclear model. only in recent years 20 ; enough experimental data on the nuclear level. spacing has been accumulated for such a comparison. However, experimental information is far from being complete.

\section{A. Termi Degeneratc Gav Model}

In an extremely simplified picture, a nucleus can be described as a Fermi degenerate gas ${ }^{21}$. Thus, we can ignore the Coulomb effects i.e., we can.consider the protons and neutrons as just nucleons. With such a description, the problem of nuclear. level density. simply reduces to the following: in how many :ways a given excitation energy can be distributed amongst all the nucleons in a nucleus. It should be noted that we are not.concerned with a single nucleon excitation, because the nuclear level spacing, computed from such a consideration, would be constant (with respect to the excitation energy) contrary. to the experimental facts. Therefore, we are dealing with a statistical description of the nuclear level density in terms of many-particle excitations.

For the range of neutron energy of interest (to the reactor problems); the decay time of the compound nucleus is much larger than the nuclear relaxation time. As a consequence of this fact, there is no phase relation between the formation of the compound nucleus and its decay. Therefore, the decay of this system is governed by the statistical considerations. In this regard, the process may be compared to the classical process of evaporation of heated liquid drops. 
Bethe $^{21}$ gives for the level density $\rho(E)$

$$
\rho(E)=C \cdot e^{2(a E)^{1 / 2}}
$$

where $C$ is a numerical constant and $E$ is the excitation energy. The simplified theory predicts that the level density parameter a is proportional to the mass number $A$.

Weinberg and Blatt ${ }^{22}$ proposed Eq. :(B-2)

$$
\rho(E)=C \cdot e^{2\left(a E^{8}\right)^{1 / 2}}
$$

where

$$
\bar{E}^{\mathrm{r}}=\mathrm{E}-\dot{\delta} /[1-\exp [-a(\mathrm{E}-\dot{\delta})]]
$$

with $\delta$ equal to the pairing energy of a nucleus. It should be pointed out that the level density is essentially governed by $2 \sqrt{6 \mathrm{E}}$ at high excitation energies. However, some difficulties arise when $\mathrm{E} \rightarrow \mathrm{O}$. Lang and Le Couteour ${ }^{23}$ have modified the analytical expression so as to get a finite result for the, case $\mathrm{E} \rightarrow \mathrm{O}$ 。

It must be noted that the single Fermion states are also characterized by the projection of the angular momentum, $m$, on the $z$ axis. The projection of the total angular momentum, $M$, on the $z$ axis of the system is the sum of projections of the $n$ excited particles. The application of random coupling to the problem gives essentially a Gaussian distribution exp $\left[-M^{2} / \alpha^{2} \sigma^{?}\right]$, where the mean square deviation

$$
\sigma^{2}=n<\stackrel{2}{m}>
$$

The density of states of a certain $M$ is therefore given by

$$
\rho(E, M)=\rho(E) \frac{e^{-M^{2} / 2 \sigma^{2}}}{\sqrt{2 \pi \sigma^{2}}}
$$


which gives

$$
\rho(E, y)=\frac{(2 j+1) \cdot \rho(E)}{2 \sqrt{2 \pi} \sigma^{3}} e^{-\frac{j(j+1)}{2 \sigma^{2}}}
$$

The approximation used in Eq. $(B-3)$ is excellent for a very large range of $j$. The value of $\sigma$ lies between 2 and 5 . Erieson $^{24}$ gives $6 \sim 4$ for nuclides around atomic weight:55. $\sigma^{2}$ is also related to the nuclear moment of inertia, $I$, and the nuclear temperature, $t$

$$
\sigma^{2}=\operatorname{Tt}^{2} \mathrm{n}^{2}
$$

Since $I \sim A^{5 / 3}$ and $t \sim A^{-1 / 2}, \sigma \sim A^{1 / 2}$ However, not enough experimental information is avallable to verify this theoretical prediction. It should be noted that the nuclear temperature is defined as follows

$$
\frac{1}{t}=\frac{d}{d E} \cdot \ln \rho(E)
$$

\section{B. Newton's Model}

It was observed that the important region of a single particle level spacing is near the Fermi. surface. Newton ${ }^{25}$ assumes that $\bar{g}_{0}(t)$, the average single particle (nuclear) level density, at a temperature $t$, is a slowly varying function of the temperature. Whereas no effect of magic nuclei: was considered in the treatment described in the preceding section, Newton considers these effects by.: calculating $E(E)$ from $F_{1} .(B-4)$.

$$
g(E):=2 \alpha A^{2 / 3}\left(j_{N}+j_{z}+1\right)
$$

$j_{g}$ and $j_{\mathbb{N}}$ are the spins of the proton and neutron shell model states,. which correspond to the atomic number $A(\Rightarrow N+z){ }_{0} \alpha$ is adjusted by an empirical fit to the experimental data; and $j_{\mathbb{N}}$ and $j_{z}$ are calculated from Kinkenberg's scheme 26 The level density $\rho(E, j)$ is related to $D(E, j)$, the level spacing by the following

$$
\rho(E, \underline{j})=1 / D(E ; j)
$$


Newton gives

$$
D=C(2 j+1)^{-1} A\left[G_{1} G_{2}\right]^{1 / 2}(2 u+3 t)^{2} \exp \left\{-2\left(\pi^{2} G u / 6\right)^{1 / 2}\right\}
$$

where: $\quad G_{1}=\alpha\left(2 \dot{\mathrm{j}}_{\dot{\mathrm{N}}}+1\right) \mathrm{A}^{2 / 3}$

$$
\begin{aligned}
& G_{2}=\alpha\left(2 \bar{j}_{\dot{z}}+1\right) A^{2 / 3} \\
& G=2 \alpha\left(\bar{j}_{N}+\bar{j}_{z}+1\right) A^{2 / 3}
\end{aligned}
$$

The temperature $t$ is given by Eq。(6):

$$
t=\left(6 u / \pi^{2} G\right)^{1 / 2}
$$

The effective $\bar{j}_{z}$ and $\bar{j}_{\mathrm{N}}$ are given in Tables B-I and B-II. $u$ is the effective excitation energy.

$$
u=E-\delta
$$

where $E$-may correspond to the energy of the incident neutron in the process of inelastic scattering and $\delta$ is the pairing energy.

For $A>40, \delta \approx 0.82(4-A / 100) \mathrm{Mev} .8 \mathrm{may}$ be taken to be (approximately) as 3.5 Mev for $A<40$, or $\delta \approx A-1 / 2$ Mev, as recomended by Green and Edwards ${ }^{27}$.

Newton was able to fit the experimental data on level spacing (including the magic nuclei) within an uncertainty of a factor of three.

Ericson ${ }^{24}$ followed Newton's method and found a fit given by Eq. (B-8):

$$
o(u, j)=c \frac{j(j+1)}{u^{2}} \exp \left[-\frac{j(j+1)}{2 \sigma^{2}}\right] \exp 2[a u]^{1 / 2}
$$

where $u$ is the effective excitation energy. The value of the parameter a was found to be

$$
a=0.056\left(\bar{j}_{z}+\bar{j}_{N}+1\right) A^{2 / 3}
$$


TABEE B-T

Effective Proton Spin Parameters

\begin{tabular}{|c|c|c|c|}
\hline z & $\begin{array}{c}\text { Effective } \\
\left(2 \overline{\mathrm{j}}_{\mathrm{z}}+1\right)\end{array}$ & $\mathrm{z}$ & $\begin{array}{c}\text { Effective } \\
\left(2 \bar{j}_{\mathrm{z}}+1\right)\end{array}$ \\
\hline 1 & 2.0 & 49 & 9.6 \\
\hline 2 & 2.67 & 50 & 9.2 \\
\hline 3 & 3.33 & 51 & 8.8 \\
\hline $4=5$ & 4.0 & 52 & 8.4 \\
\hline 6 & 3.33 & $.53-56$ & 8.0 \\
\hline 7 & 2.67 & 57 & 8.2 \\
\hline 8 & 3.33 & .58 & 8.4 \\
\hline 9 & 4.67 & .59 & 8.6 \\
\hline $10-13$ & 6.0 & .60 & 8.8 \\
\hline 14 & .4 .67 & $61-64$ & 9.0 \\
\hline 15 & 3.33 & $65-74$ & 9.0 \\
\hline 16 & 2.67 & 75 & 8.0 \\
\hline 17 & 3.33 & 76 & 7.0 \\
\hline $18 \div 19$ & 4.0 & 77 & 6.0 \\
\hline 20 & 5.33 & 78 & 5.0 \\
\hline $21^{\circ}$ & 6.67 & 79 & 3.6 \\
\hline $22-27$ & 8.0 & 80 & 3.2 \\
\hline 28 & 6.67 & 81 & 4.04. \\
\hline 29 & .5 .33 & 82 & 5.6 \\
\hline $30-3 I$ & 4.0 & 83 & 6.8 \\
\hline 32 & 4.67 & 81 & 8.4 \\
\hline 33 & 5.33 & $85-90$ & 10.0 \\
\hline $34-37$ & 6.0 & 91 & 9.867 \\
\hline 38 & 4.67 & 92 & 9.733 \\
\hline 39 & 3.33 & 93 & 9.6 \\
\hline 40 & 4.67 & .94 & 9.467 \\
\hline 41 & 7.33 & $95-98$ & 9.33 \\
\hline $42-48$ & 10.0 & & \\
\hline
\end{tabular}


TABLE : B-II

Effective Neutron Spin Parameters

\begin{tabular}{|c|c|c|c|c|c|}
\hline $\mathrm{N}$ & $\begin{array}{c}\text { Effective } \\
\left(2 \bar{j}_{N}+1\right)\end{array}$ & $N$ & $\begin{array}{l}\text { Effective } \\
\left(2 \hat{\mathrm{j}}_{\mathrm{N}}+1\right)\end{array}$ & $\mathrm{N}$ & $\begin{array}{c}\text { Effective } \\
\left(2 j_{\mathbb{N}}+1\right)\end{array}$ \\
\hline 1 & 2.0 & $42 \div 48$ & 10.0 & $93-100$ & .10 .0 \\
\hline 2 & 2.67 & 49 & 9.2 & $10 i-106$ & 10.0 \\
\hline 3 & $3 \cdot 33$ & .50 & 8.4 & $107-118$ & 10.0 \\
\hline $4-5$ & 4.0 & 51 & $7: 6$ & 119 & 8.8 \\
\hline 6 & $3 \cdot 33$ & 52 & $6: 8$ & 120 & 7.6 \\
\hline 7 & 2.67 & $53-54$ & $6: 0$ & 121 & $6: 4$ \\
\hline 8 & 3.33 & 55 & 6.8 & 122 & 5.2 \\
\hline 9 & 4.67 & 56 & $7: 6$ & 123 & $3: 6 \ldots \ldots$ \\
\hline $10-13$ & 6.0 & 57 & 8.4 & 124 & 3.2 \\
\hline 211 & 1.67 & 50 & $\dot{y} \cdot \ddot{z}$ & 125 & 4.4 \\
\hline .15 & $3 \cdot 33$ & $59-64$ & 10.0 & 126 & 5.6 \\
\hline 16 & 2.67 & $.65-74$ & 10.0 & 127 & 6.8 \\
\hline 17 & $3 \cdot 33$ & 75 & 8.8 & 128 & 8.4 \\
\hline $18-19$ & 4.0 & .6 & $7: 6$ & $129-134$ & 10.0 \\
\hline 20 & $5 \cdot 33$ & 77 & 6.4 & 135 & 10.4 \\
\hline 21 & 6.67 & 78 & 5.2 & 136 & 10.8 \\
\hline $22-27$ & 8.0 & 79 & $3 \cdot 6$ & 137 & 11.2 \\
\hline 28 & $6: 67$ & 80 & $3 . .2$ & 138 & .11 .6 \\
\hline$: 29$ & 5.33 & 81 & 4.0 & $139-148$ & 12.0 \\
\hline $30-31$ & 4.0 & 82 & 4..8 & & \\
\hline 32 & 4.67 & 83 & $5: 6$ & & \\
\hline 33 & 5.33 & 84 & $6: 8$ & & \\
\hline $34-37$ & 6.0 & $85-88$ & 8.0 & & \\
\hline 38 & 4.67 & 89 & 8.4 & & $\ldots$ \\
\hline 39 & 3.33 & 90 & 8.8 & & \\
\hline 40 & 4.67 & 9.1 & 9.2 & & \\
\hline 41 & $7 \cdot 33$ & 92 & 9.6 & & \\
\hline
\end{tabular}


As before, $\bar{j}_{\mathrm{Z}}$ and $\bar{j}_{\mathrm{N}}$, are given in Tables $B-I$ and $B-I I$. a is plotted versus. the mass number in Fig. B-2. The solid curve corresponds to $\mathrm{Eq}_{0} \cdot(\mathrm{B}-\mathrm{9})$.

\section{Cameron's Method}

Whereas Newton tried to find a thedretical fit to the experimental data. on the nuclear level density and explained the general variation with the shell structure by. using Klinkenberg's model, cameron ${ }^{28}$ considers these effects by. a semiempirical atomic mass formula. The expression for the level density, as given by Cameron, is very complicated for calculation purposes.

$$
D(u, j) \Rightarrow \frac{3.26 \cdot 10^{5}}{(2 j+1)}\left[G_{z} G_{N V} G^{3} A^{2}\left(\frac{\pi^{2}}{3} G t-\frac{3}{2}\right) \cdot t^{7}\right]^{1 / 2} \exp -\frac{\pi^{2} G t}{3}
$$

where

$$
t=\frac{3}{\pi^{2} G}\left[\frac{3}{2}+\left(\frac{9}{4}+\frac{2 \pi^{2} G u}{3}\right)^{1 / 2}\right]
$$

and

$$
\begin{aligned}
G_{k}(t) & =\frac{a_{0}(k)+a_{1}(k) t+a_{2}(k) t^{2}+a_{3}(k) t^{3}+a_{4}(k) t^{4}}{1+t+t^{2}+t+t^{3}+t^{4}} \\
G & =G_{N}+G_{z}
\end{aligned}
$$

In Eq. (B-12), k stands for $N$ or z. Cameron fitted the fine coefficients namely $a_{0}(k)$-.--; $a_{4}(k)$ by a least square fit. to 15 values of $G_{k}(t)$. These coefficients are tabulated for $10 \leq z \leq 109$ and $10 \leq \mathbb{N} \leq 189$. However, it must be noted that for $G$ and $t$ to be determined simultaneously some iterative procedure is to be followed cameron recommends Eq. $(B-14)$

$$
G(t)_{\text {initial }}=a_{4}(z)+a_{4}(N)
$$

Nëar the closed shells it is often better to choose an initial value of about one half of the amount given by $\mathrm{Eg}_{0}(\mathrm{~B}-14)$.: 


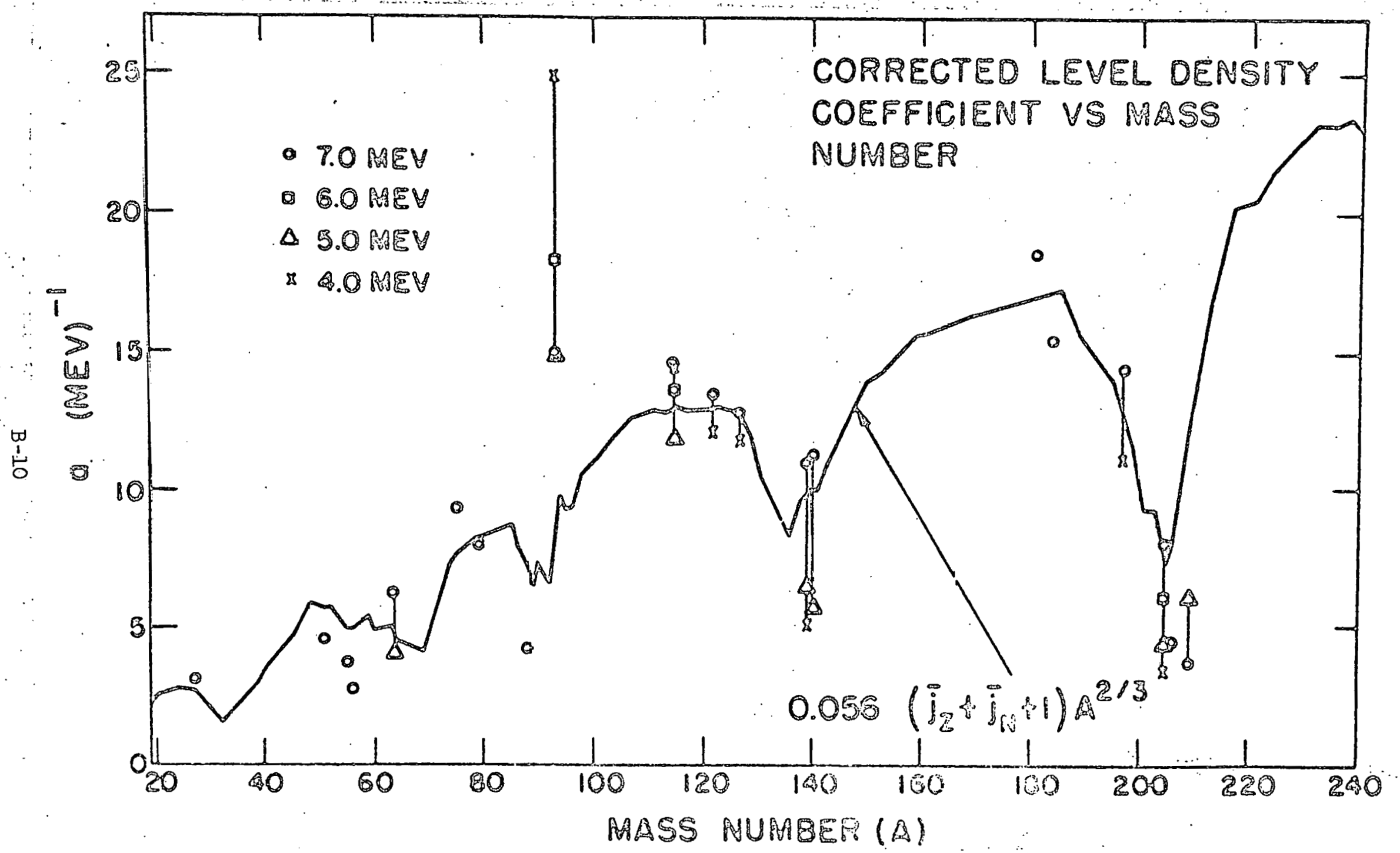

Fig. B-II The Parameter a Versus Mass Number (See Eqs. B-8 and B-9 of the text, (after Ericson).) 
The root mean square error for Cameron's model is 1.74 in contrast to a corresponding value of 3 obtained from Newton's model. It may be noted that the Newton's method corresponds to a ratio of the moment of inertia to the rigid body value of 0.13 in contrast to the corresponding ratio by Cameron's model of 0.03 . Part of the reason for such a reduction is that the cut-off term in angular momentum is not justified.

\section{DISCUSSION AND CONCLUSIONS}

In the first place, most of the existing experimental data on the nuclear level density can be explained by the various theoretical models. For example, it should be noted that the Newton's method (as well as Cameron's model) can effectively explain the large variation of the nuclear level spacings with the mass number A. However, a great caution is to be exercised in particular cases especialIy when accurate computations of the inelastic scattering matrix are desirable. Though the nuclear level density has generally been taken to be proportional to $\exp [2 \text { a } E]^{1 / 2}$, yet this is valia only for high excitation energies (neutron energy above 9 Mev or so).

The most convenient and quite accurate treatment is due to Ericson, who followed the basic model of Newton. The relevant expression is given in Eq. (B-8). However, Eq. (B-1) can be profitably used whenever an accurate determination of the parameter $a$ is available from a detailed analysis of the experimental data in particular cases. 